\title{
A systematic review of TMS and neurophysiological biometrics in patients with schizophrenia
}

\author{
Meng di Hou, MSc*; Viviana Santoro, MSc*; Andrea Biondi, MSc; \\ Sukhi S. Shergill, MBBS, PhD ${ }^{\dagger}$ Isabella Premoli, $\mathrm{PhD}^{\dagger}$
}

\begin{abstract}
Background: Transcranial magnetic stimulation can be combined with electromyography (TMS-EMG) and electroencephalography (TMS-EEG) to evaluate the excitatory and inhibitory functions of the cerebral cortex in a standardized manner. It has been postulated that schizophrenia is a disorder of functional neural connectivity underpinned by a relative imbalance of excitation and inhibition. The aim of this review was to provide a comprehensive overview of TMS-EMG and TMS-EEG research in schizophrenia, focused on excitation or inhibition, connectivity, motor cortical plasticity and the effect of antipsychotic medications, symptom severity and illness duration on TMS-EMG and TMS-EEG indices. Methods: We searched PsycINFO, Embase and Medline, from database inception to April 2020, for studies that included TMS outcomes in patients with schizophrenia. We used the following combination of search terms: transcranial magnetic stimulation OR tms AND interneurons OR glutamic acid OR gamma aminobutyric acid OR neural inhibition OR pyramidal neurons OR excita* OR inhibit* OR GABA* OR glutam* OR E-I balance OR excitation-inhibition balance AND schizoaffective disorder* OR Schizophrenia OR schizophreni*. Results: TMS-EMG and TMS-EEG measurements revealed deficits in excitation or inhibition, functional connectivity and motor cortical plasticity in patients with schizophrenia. Increased duration of the cortical silent period (a TMS-EMG marker of $\gamma$-aminobutyric acid B receptor activity) with clozapine was a relatively consistent finding. Limitations: Most of the studies used patients with chronic schizophrenia and medicated patients, employed cross-sectional group comparisons and had small sample sizes. Conclusion: TMS-EMG and TMS-EEG offer an opportunity to develop a novel and improved understanding of the physiologic processes that underlie schizophrenia and to assess the therapeutic effect of antipsychotic medications. In the future, these techniques may also help predict disease progression and further our understanding of the excitatory/inhibitory balance and its implications for mechanisms that underlie treatment-resistant schizophrenia.
\end{abstract}

\section{Introduction}

Schizophrenia is a neuropsychiatric illness that affects nearly $1 \%$ of the population. ${ }^{1}$ It is characterized by 3 main clusters of symptoms: positive symptoms (e.g., delusions and hallucinations), negative symptoms (e.g., lack of motivation, reduction in spontaneous speech and social withdrawal) and cognitive dysfunction. ${ }^{2}$ The pathophysiology and cause of schizophrenia remain unclear; schizophrenia is partly considered a genetically mediated disorder in which etiologic and pathogenic factors occur before the onset of illness, altering the normal development of specific neural circuits and conferring a degree of vulnerability. ${ }^{3-5}$ Abnormal dopaminergic signalling - involving an increase of dopamine synthesis, release and resting-state synaptic concentrations - has been the leading theory in the pathophysiology of schizophrenia. All current antipsychotics act as dopamine receptor antagonists and demonstrate their greatest efficacy in treating positive symptoms, ${ }^{2,6}$ with limited (if any) benefit for negative symptoms and cognitive deficits. Up to onethird of patients treated with antipsychotic medication do not respond to treatment, even with adequate dopaminergic receptor blockade. ${ }^{7}$ This has led to explorations of the role of glutamatergic ${ }^{8}$ and $\gamma$-aminobutyric acid (GABA)-ergic neurotransmission, which can both cause an altered excitation/ inhibition (E/I) balance. ${ }^{6,9,10}$ This assumption has been confirmed by postmortem studies showing a reduction in glutamic acid decarboxylase 67 (GAD67), a synthetic enzyme for GABA, in parvalbumin GABAergic interneurons. In addition, several pharmacological and animal models have

Correspondence to: M. Hou, Floor 7, Department of Psychosis Studies, Institute of Psychiatry, Psychology and Neuroscience (loPPN), 16 De Crespigny Park, London, SE5 8AF; meng_di.hou@ kcl.ac.uk

*These authors have joint first authorship.

†These authors contributed equally to this work.

Submitted Jan. 12, 2021; Revised Aug. 6, 2021; Accepted Sep. 6, 2021

Cite as: J Psychiatry Neurosci 2021 December 21;46(6). doi: 10.1503/jpn.210006 
shown that a deficit in GAD67 is associated with N-methylD-aspartate (NMDA) receptor hypofunction in these neurons, leading to disinhibition of pyramidal cells. ${ }^{11-16}$ This E/I balance offers a mechanism by which the different neurotransmitter systems can influence (dysfunctional) perceptual and cognitive processing, leading to neural dysconnectivity and the development of psychotic symptoms.

Transcranial magnetic stimulation combined with electromyography (TMS-EMG) has emerged as a noninvasive neurophysiological tool for probing the function of inhibitory and excitatory neural elements in a standard manner, and for exploring their connectivity in different neuropsychiatric disorders. ${ }^{17}$ Single-pulse TMS over the human primary motor cortex induces a motor evoked potential (MEP), which is defined as the reaction of the peripheral muscle measured by EMG recordings. The amplitude of the MEP reflects the activation of corticospinal neurons through a network regulated by glutamatergic, GABAergic and neuromodulating neurotransmitters. ${ }^{18,19}$ The minimum amount of stimulation intensity necessary to elicit a small MEP defines the threshold that can be measured at rest (resting motor threshold; RMT) or during a small voluntary contraction (active motor threshold; AMT). Because motor thresholds are increased after the administration of voltagegated sodium channel blockers, they are considered to reflect the activity of axons and their excitatory synaptic contacts with the corticospinal neurons. ${ }^{19-23}$

Inhibitory processes can be indexed by 3 paradigms: cortical silent period (CSP), short-interval intracortical inhibition (SICI) and long-interval intracortical inhibition (LICI). CSP is a single-pulse protocol defined as the TMS-induced interruption of voluntary myographic activity. The early segment of the CSP is related to spinal inhibition, and the later segment is hypothesized to be of motor cortical origin and mediated by GABA-B receptors. ${ }^{24-26}$ Paired-pulse TMS protocols consist of 2 TMS pulses: a conditioning stimulus followed by a test stimulus. Depending on the interstimulus interval and the intensity of the conditioning stimulus pulse, these paradigms can activate specific intracortical inhibitory or facilitatory mechanisms. ${ }^{27}$ Pharmacological studies in healthy volunteers show that GABA-A receptor-positive modulators increase SICI and GABA-B receptor agonists increase LICI, indicating a link with GABA-A and GABA-B receptor-mediated inhibition, respectively. 28,29

Cortical excitability can be indexed by 2 other pairedpulse paradigms: intracortical facilitation (ICF) and shortinterval intracortical facilitation (or I-wave). ICF is modulated by NMDA receptors and GABA-A receptors; ${ }^{30-32}$ short-interval intracortical facilitation reflects glutamatergic activity likely mediated by non-NMDA receptors. Several studies have also found that short-interval intracortical facilitation is controlled by cortical inhibition, because allosteric modulators of GABA-A receptors decrease this parameter. ${ }^{33}$ TMS-EMG can be used to measure the level of connectivity between the 2 motor cortices using a protocol named transcallosal inhibition. ${ }^{34,35}$

TMS has been combined with simultaneous electroencephalography (EEG) to obtain a more detailed profile response of the human brain to perturbations on a time scale of milliseconds. ${ }^{36-38}$ TMS-EEG can measure the strength of inhibitory and excitatory neural responses of the targeted brain area and at distant sites by measuring connectivity patterns in health and in several pathological conditions. ${ }^{39}$ EEG responses can be interrogated in the time domain and in the frequency domain. Time-locked responses after stimulation of the motor cortex are called TMS-evoked EEG potentials and are a series of positive $(\mathrm{P})$ and negative $(\mathrm{N})$ deflections at approximately 25 (P25), 45 (N45), 100 (N100) and 180 (P180) milliseconds after stimulation. Specific components of TMSevoked EEG potentials have been linked to cortical excitatory and inhibitory processes by applying pharmacological interventions in healthy volunteers. For example, the N45 and N100 amplitudes have been associated with inhibition mediated by the GABA-A and GABA-B receptors, respectively. ${ }^{40,41}$ Time-frequency decomposition of the TMS-EEG signal results in TMS-induced oscillations that represent information that is not necessarily phase-locked to the stimulus. Singlepulse TMS over the primary motor cortex elicits a specific pattern consisting of an early increase of $\theta-, \alpha-$ and $\beta$-band power, followed by a $\beta$ suppression and a final $\beta$ rebound. Pharmacological studies have shown that early $\alpha$ synchronization is increased by GABA-A-ergic drugs and decreased by GABA-B-ergic drugs, whereas $\beta$ suppression is increased by GABA-A-ergic and GABA-B-ergic drugs. ${ }^{42}$

The pathophysiology of schizophrenia has been associated with abnormalities in brain plasticity, which refers to the ability of the brain to adapt in response to experience. ${ }^{43}$ TMS and other noninvasive stimulation techniques are used to elicit plastic changes in synaptic organization, interfering with the function of specific cortical areas by inducing long-term potentiation or long-term depression. ${ }^{44-47}$ For example, transcranial direct current stimulation (tDCS) is a neuromodulation technique that induces changes in cortical excitability by applying a small current over the scalp. This technique modulates the activity of spontaneous neurons by tonic depolarization (anodal tDCS) or hyperpolarization (cathodal tDCS) of their membrane, inducing long-lasting changes in neuronal firing rates. ${ }^{48-51}$ The paired associative stimulation protocol refers to a paradigm that consists of repetitive lowfrequency median nerve stimulation combined with TMS over the contralateral motor cortex. Paired associative stimulation induces changes in the size of MEPs that depend on the exact time interval between the afferent and the magnetic pulse during the intervention..$^{52}$

Despite growing interest in this area, the current literature lacks a systematic review summarizing how TMS combined with EEG and EMG can provide a robust functional index of E/I alteration in schizophrenia. Our purpose was to provide such a systematic review, showing how biometrics related to TMS-EMG and TMS-EEG measure specific cortical processes of inhibition, excitation and connectivity in schizophrenia, and describing their relationship with clinical outcomes. We aimed to offer insight into the potential of a multimodal, noninvasive approach to brain stimulation to explore the neurophysiological mechanisms involved in schizophrenia. 


\section{Methods}

\section{Search strategy}

We performed a systematic literature search using Medline, Embase and PsycINFO. We used the following combination of search terms: transcranial magnetic stimulation OR tms AND interneurons OR glutamic acid OR gamma aminobutyric acid OR neural inhibition OR pyramidal neurons OR excita* OR inhibit* OR GABA* OR glutam* OR E-I balance OR excitation-inhibition balance AND schizoaffective disorder* OR Schizophrenia OR schizophreni*. The search yielded a total of 665 results (see Appendix 1, available at jpn.ca, for details of the search terms for each database).
The literature search was conducted independently by 2 researchers (V.S. and M.D.H.) in line with Preferred Reporting Items for Systematic Reviews and Meta-Analyses (PRISMA) guidelines. The researchers screened titles and abstracts; eliminated duplicates; read the full texts of the remaining papers; selected the papers that met the inclusion criteria; and extracted relevant data. They also manually searched the references of the selected studies for additional articles to include. Discrepancies or disagreement were resolved through discussion. A PRISMA flow diagram is shown in Figure 1.

\section{Selection criteria}

Based on our research question, we included primary research articles that met the following criteria: diagnoses of
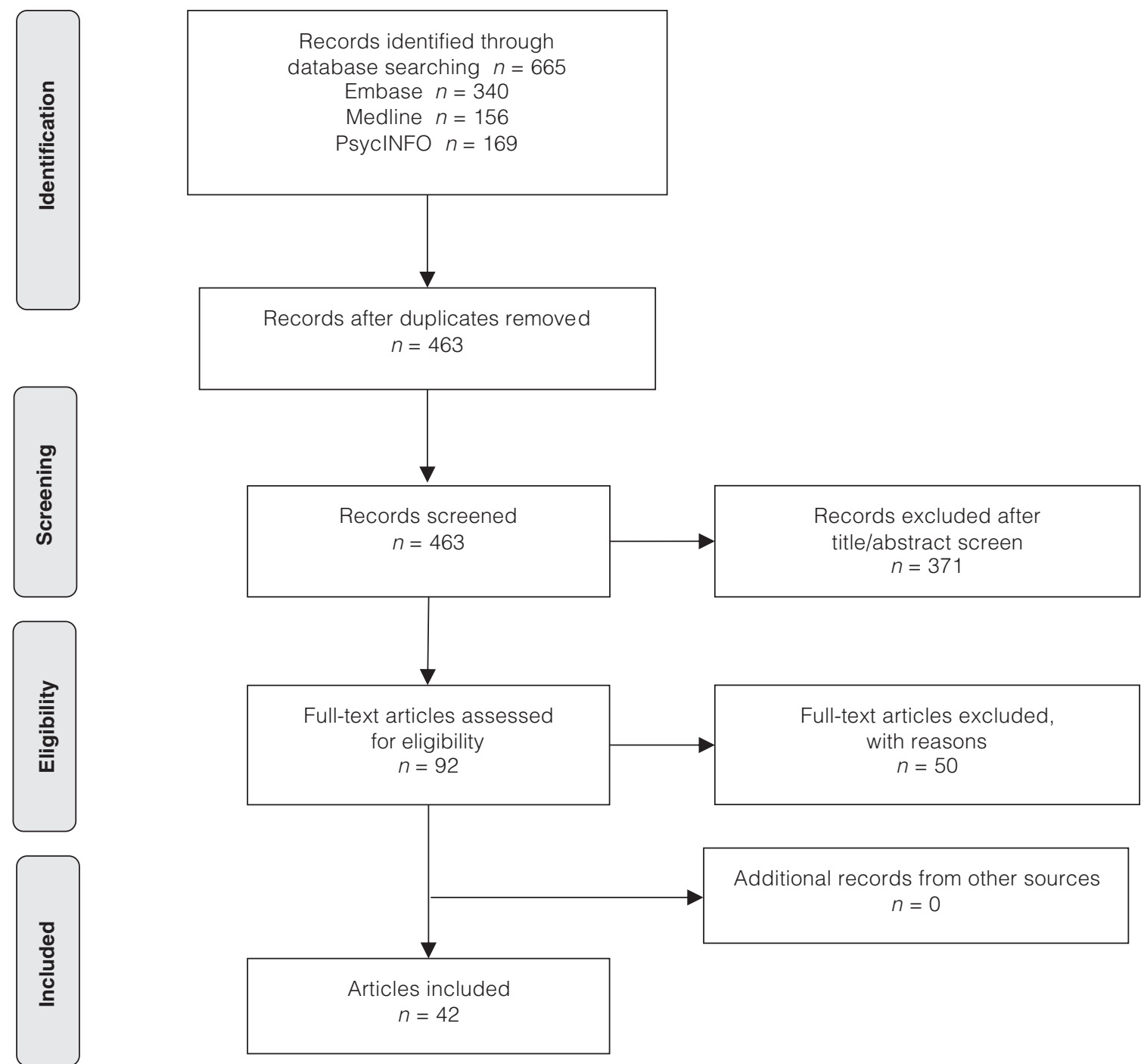

Full-text articles excluded, with reasons $n=50$

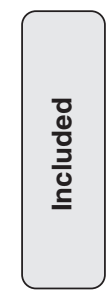

Additional records from other sources $n=0$

Figure 1: PRISMA flow diagram of included studies. PRISMA = Preferred Reporting Items for Systematic Reviews and Meta-Analyses. 
schizophrenia or schizoaffective disorder made using standardized diagnostic criteria; participants between 18 and 65 years old; participants without a documented substance abuse disorder; studies that included TMS outcomes.

We included only studies published in English or available in translation. We excluded reviews, conference abstracts, opinion papers, cases series or case reports.

\section{Data extraction}

We recorded the following variables from the full-text articles: authors, year of publication, study population, clinical characteristics, medications, TMS protocol, task, stimulation site and measures of cortical excitability. All outcomesrelated data were extracted independently by the 2 researchers (V.S. and M.D.H.).

\section{Risk of bias assessment}

We assessed risk of bias using an adapted version of the Risk of Bias Assessment for Non-Randomized Studies (RoBANS) tool, which evaluates the following factors: selection of participants, confounding variables, measurement of exposure, blinding of outcome assessments, incomplete outcome data and selective outcome reporting. ${ }^{53}$ See Appendix 1 for the RoBANS scale used.

\section{Results}

"Patients" refers to patients with schizophrenia or schizoaffective disorder unless specified otherwise. Specifically, 1 study $^{54}$ evaluated people at ultra-high risk of developing psychosis. According to the authors of that study, "ultrahigh risk" is defined as a period of imminent risk of developing psychosis in adolescents and young adults, ${ }^{55}$ and participants met the criteria for an at-risk mental state as defined by the Structured Interview for Prodromal Syndromes (SIPS) ${ }^{56}$ and a scale for prodromal syndromes. ${ }^{57}$ Another study ${ }^{58}$ used participants from a randomized controlled trial on the secondary prevention of schizophrenia. ${ }^{59}$ Participants at risk in this study fulfilled the criteria for at least 1 of the following groups: attenuated positive symptoms; brief limited intermittent psychotic symptoms; predictive basic symptoms; family risk for psychosis plus reduced functioning (see Bechdolf and colleagues ${ }^{59}$ ). Two studies also tested patients with recent-onset schizophrenia. Takahashi and colleagues ${ }^{60}$ followed patients with schizophrenia (DSM-IV) and a duration of illness of less than 3 years. Hasan and colleagues ${ }^{58}$ compared patients with recent-onset schizophrenia (duration of psychosis less than 2 years and a single psychotic episode that lasted for at least 1 month) with patients who had multi-episode schizophrenia (more than 2 psychotic episodes, at least 1 relapse and a duration of psychosis of more than 2 years).

Details of the studies that reported TMS-EMG and TMSEEG outcomes and patient characteristics are shown in Appendix 1, Table S1 and Table S2.

\section{TMS-EMG in schizophrenia}

The TMS-EMG parameters assessed in the included studies are shown in Table 1. Summaries of the protocols and speculated mechanisms for each parameter were taken from the review by Ziemann and colleagues. ${ }^{19}$ Table 2 provides a brief overview of the findings for each TMS-EMG measure.

\section{Resting motor threshold and active motor threshold}

In 19 of 20 studies, all or most of the patients were medicated. In 15 of the 19, no significant differences in RMT were reported between patients and healthy controls. ${ }^{54,60,78-90}$ This lack of difference in RMT was observed in patients with chronic schizophrenia but also extended to medication-naive or minimally treated ( $<1$ month) patients with first-episode psychosis. ${ }^{91}$

Lower RMT values (indicating increased corticospinal excitability) were reported in only 1 study of medicated patients with schizophrenia, but also in patients with major depressive disorder and manic disorder.$^{92}$ In contrast, Bridgman and colleagues ${ }^{93}$ and Soubasi and colleagues ${ }^{94}$ reported elevated RMT values in medicated patients with chronic schizophrenia relative to healthy controls, indicating decreased corticospinal excitability. Finally, a study that compared medicated patients with recent-onset schizophrenia (i.e., with a single psychotic episode), medicated patients with multiepisode schizophrenia (with more than 1 relapse of the illness) and healthy controls showed high RMT values in the pooled patient groups. ${ }^{45}$

With respect to AMT, our search found only 2 studies that showed nonsignificant changes between patients with chronic schizophrenia and healthy controls ${ }^{84,86}$ or healthy siblings. ${ }^{86}$ Both studies used mostly medicated patients.

In summary, studies of AMT are relatively rare, but several studies explored RMT in patients with schizophrenia, and most of the results showed no significant effect in either direction. Interpretation of the few other studies that demonstrated increased and decreased RMT may reflect disease heterogeneity or the persisting effects of medication. ${ }^{97}$

\section{Amplitude of motor evoked potentials}

Amplitudes of MEPs did not differ between patients with chronic schizophrenia (medicated and unmedicated) and healthy controls, regardless of whether the hand area of the primary motor cortex was stimulated with a suprathreshold intensity, ${ }^{87}$ at $120 \% \mathrm{RMT}^{79,82}$ or with the lowest stimulus intensity required to produce maximum MEPs. ${ }^{94}$ Similarly, MEPs did not differ between patients (most of whom were medicated) and healthy controls when the motor cortex was stimulated with an intensity intended to elicit an average MEP of $1 \mathrm{mV}$ amplitude (SI-1mV), in either intensity ${ }^{76,89,97,99}$ or amplitude. ${ }^{58,89}$

Similarly, no difference in SI-1mV MEPs was found among patients with recent-onset schizophrenia, patients with multiepisode schizophrenia, people at ultra-high risk of psychosis or healthy controls. ${ }^{45}$ However, 1 study found that the SI- $1 \mathrm{mV}$ MEP was significantly smaller in medicated inpatients with chronic schizophrenia than in healthy controls. ${ }^{54}$ Furthermore, Hasan and colleagues ${ }^{58}$ found smaller SI-1mV MEPs in people 
at risk of developing psychosis compared to patients with first-episode schizophrenia and healthy controls. These results suggest that further studies are needed to investigate deficits in cortical excitability in the early stages of psychosis.

Several studies also used the amplitude of MEPs as a TMS biometric for assessing differences in clinical abnormalities of motor function in psychiatric disorders. Chroni and colleagues $^{92}$ and Reid and colleagues ${ }^{88}$ applied TMS before and after a nonfatiguing exercise of the target hand muscle, and then calculated the MEP after exercise facilitation (expressed as a percentage of the baseline ratio) in medicated patients with chronic schizophrenia. The results were mixed: in the study by Chroni and colleagues, ${ }^{92}$ patients showed reduced MEPs after exercise facilitation compared to controls, suggesting impaired cortical excitability, but Reid and colleagues $^{88}$ found that patients with schizophrenia had higher pre-exercise MEPs compared to healthy controls.

Finally, 2 studies investigated the onset latency of MEPs, and the results were inconclusive: Boroojerdi and colleagues ${ }^{80}$ found no differences between medicated patients with chronic schizophrenia and healthy controls when using a stimulus intensity of $50 \%$ above the RMT, but Soubasi and

Table 1: TMS-EMG measures

\begin{tabular}{|c|c|c|c|c|}
\hline Measure & Protocol & Speculated mechanism & Pharmacological evidence & References \\
\hline \multicolumn{5}{|l|}{ Single-pulse TMS } \\
\hline $\begin{array}{l}\text { Resting motor threshold } \\
\text { and active motor } \\
\text { threshold }\end{array}$ & $\begin{array}{l}\text { Minimum TMS intensity to } \\
\text { elicit an MEP with (usually) } \\
50 \mu \mathrm{V} \text { peak-to-peak } \\
\text { amplitude in the target } \\
\text { muscle, either at rest (RMT) } \\
\text { or during voluntary } \\
\text { contraction (AMT) }\end{array}$ & $\begin{array}{l}\text { Voltage-gated, sodium- } \\
\text { channel-mediated neuronal } \\
\text { membrane excitability }\end{array}$ & $\begin{array}{l}\text { Increased by voltage-gated } \\
\text { sodium channel blockers (e.g., } \\
\text { carbamazepine, phenytoin and } \\
\text { lamotrigine); decreased } \\
\text { by ketamine }\end{array}$ & $\begin{array}{l}\text { Lang et al. }{ }^{61}(2013) \\
\text { Menzler et al. }{ }^{62}(2014) \\
\text { Ziemann et al. }{ }^{63}(1996)\end{array}$ \\
\hline $\begin{array}{l}\text { Amplitude of motor } \\
\text { evoked potentials }\end{array}$ & $\begin{array}{l}\text { Evoked by stimulus intensity } \\
\text { above the motor threshold }\end{array}$ & $\begin{array}{l}\text { Trans-synaptic activation of } \\
\text { corticospinal neurons } \\
\text { regulated by glutamatergic, } \\
\text { GABAergic and } \\
\text { neuromodulating } \\
\text { neurotransmitters }\end{array}$ & $\begin{array}{l}\text { Increased by ketamine, } \\
\text { noradrenaline agonists (e.g., } \\
\text { methylphenidate) and 5-HT } \\
\text { agonists (e.g., sertraline); } \\
\text { decreased by positive allosteric } \\
\text { modulators of GABA receptors } \\
\text { (e.g., lorazepam) }\end{array}$ & $\begin{array}{l}\text { Ilic et al. }{ }^{64}(2003) \\
\text { Gerdelat-Mas et al. }{ }^{65}(2005) \\
\text { Paulus et al. }{ }^{66}(2008) \\
\text { Boroojerdi et al. }{ }^{30}(2001) \\
\text { Di Lazzaro et al. }{ }^{67}(2000)\end{array}$ \\
\hline $\begin{array}{l}\text { Duration of cortical silent } \\
\text { period }\end{array}$ & $\begin{array}{l}\text { Duration of TMS-induced } \\
\text { interruption in voluntary } \\
\text { EMG activity of the } \\
\text { target muscle }\end{array}$ & $\begin{array}{l}\text { Motor cortical inhibition } \\
\text { mediated by activation of } \\
\text { GABA-A receptors (short } \\
\text { CSPs) or GABA-B } \\
\text { receptors (long CSPs) }\end{array}$ & $\begin{array}{l}\text { Increased by benzodiazepines } \\
\text { (short CSPS) and baclofen } \\
\text { (specific GABA-B receptor } \\
\text { agonist) }\end{array}$ & $\begin{array}{l}\text { Inghilleri et al. }{ }^{68}(1996) \\
\text { Siebner et al. }{ }^{69}(1998)\end{array}$ \\
\hline $\begin{array}{l}\text { Short-latency afferent } \\
\text { inhibition }\end{array}$ & $\begin{array}{l}\text { Conditioning afferent } \\
\text { electrical stimulus to the } \\
\text { median or ulnar nerve at the } \\
\text { wrist precedes TMS of the } \\
\text { contralateral motor cortex by } \\
\text { roughly } 20 \text { ms }\end{array}$ & $\begin{array}{l}\text { Physiologic marker of the } \\
\text { integrity and excitability of } \\
\text { central cholinergic } \\
\text { pathways }\end{array}$ & $\begin{array}{l}\text { Increased by } \\
\text { acetylcholinesterase inhibitors } \\
\text { and nicotine; decreased by } \\
\text { scopolamine (acetylcholine } \\
\text { antagonist) and lorazepam } \\
\text { (benzodiazepine) }\end{array}$ & $\begin{array}{l}\text { Di Lazzaro et al. }{ }^{70}(2005) \\
\text { Di Lazzaro et al. }{ }^{71}(2005) \\
\text { Di Lazzaro et al. } .^{67}(2000)\end{array}$ \\
\hline Transcallosal inhibition & $\begin{array}{l}\text { Single TMS pulses over the } \\
\text { motor cortex ipsilateral to the } \\
\text { voluntarily contracted hand } \\
\text { muscle to induce a silent } \\
\text { period in EMG activity }\end{array}$ & $\begin{array}{l}\text { Duration of the silent period } \\
\text { is thought to reflect the } \\
\text { functioning of the corpus } \\
\text { callosum and an inhibitory } \\
\text { system in the contralateral } \\
\text { motor cortex }\end{array}$ & & $\begin{array}{l}\text { Ferbert et al. }{ }^{34}(1992) \\
\text { Meyer et al. } .^{72}(1998)\end{array}$ \\
\hline \multicolumn{5}{|l|}{ Paired-pulse TMS } \\
\hline $\begin{array}{l}\text { Short-interval } \\
\text { intracortical inhibition }\end{array}$ & $\begin{array}{l}\text { Subthreshold conditioning } \\
\text { stimulus precedes } \\
\text { suprathreshold test stimulus } \\
\text { by } 1 \sim 5 \mathrm{~ms}\end{array}$ & $\begin{array}{l}\text { Short-lasting inhibition in } \\
\text { regional corticospinal } \\
\text { neurons mediated by } \\
\text { GABA-A receptors } \\
\text { containing } \alpha 2 \text { or } \alpha 3 \\
\text { subunits }\end{array}$ & $\begin{array}{l}\text { Increased by benzodiazepines } \\
\text { (positive modulators at } \alpha 1, \alpha 2 \text {, } \\
\alpha 3 \text { or } \alpha 5 \text { subunits of GABA-A } \\
\text { receptors); not affected by } \\
\text { zolpidem (specific positive } \\
\text { modulator of } \alpha 1-\text { GABA-A } \\
\text { receptor) or } S 44819 \text { (selective } \\
\text { antagonist of } \alpha 5-G A B A-A \\
\text { receptor) }\end{array}$ & $\begin{array}{l}\text { Di Lazzaro et al. }{ }^{73}(2007) \\
\text { Di Lazzaro et al. }{ }^{74}(2006)\end{array}$ \\
\hline Intracortical facilitation & $\begin{array}{l}\text { Subthreshold conditioning } \\
\text { stimulus precedes } \\
\text { suprathreshold test stimulus } \\
\text { by } 7 \sim 20 \mathrm{~ms}\end{array}$ & $\begin{array}{l}\text { Net excitation of an } \\
\text { excitatory motor cortical } \\
\text { network }\end{array}$ & $\begin{array}{l}\text { Increased by noradrenergic } \\
\text { agonists; decreased by NMDA } \\
\text { receptor antagonists and } \\
\text { benzodiazepines }\end{array}$ & $\begin{array}{l}\text { Ziemann et al. }{ }^{32}(1998) \\
\text { Ziemann et al. }{ }^{75}(1996) \\
\text { Boroojerdi et al. }{ }^{30}(2001)\end{array}$ \\
\hline $\begin{array}{l}\text { Long-interval intracortical } \\
\text { inhibition }\end{array}$ & $\begin{array}{l}\text { Two suprathreshold } \\
\text { conditioning and test } \\
\text { stimuli separated by } \\
50 \sim 200 \mathrm{~ms}\end{array}$ & $\begin{array}{l}\text { GABA-B receptor-mediated } \\
\text { slow inhibitory postsynaptic } \\
\text { potentials }\end{array}$ & $\begin{array}{l}\text { Increased by baclofen, tiagabine } \\
\text { and vigabatrin }\end{array}$ & $\begin{array}{l}\text { Pierantozzi et al. }{ }^{76}(2004) \\
\text { Werhahn et al. }{ }^{77}(1999) \\
\text { McDonnell et al. }{ }^{29}(2006)\end{array}$ \\
\hline
\end{tabular}


colleagues $^{94}$ found a longer MEP latency in patients in response to the lowest stimulus intensity required to produce maximum MEPs.

Overall, MEPs did not differ significantly in patients with chronic schizophrenia, but it is possible that people in at-risk prodromal states may show lower MEPs, suggestive of impaired cortical excitability.

\section{Duration of cortical silent period}

Compared to healthy controls, medicated patients with chronic schizophrenia demonstrated no significant differences in CSP duration (an index of spinal inhibition and the relative level of GABA-B receptor-mediated inhibition) at a stimulus intensity of $140 \%$ RMT or SI- $1 \mathrm{mV}$ in 3 of the 10 studies we as- sessed. ${ }^{45,93,95}$ An additional study that investigated differences in CSP duration in medicated patients with chronic schizophrenia, healthy siblings of patients and healthy controls did not show any difference among the groups using stimulus intensities of $120 \%$ and $140 \%$ AMT. $^{86}$

However, CSP duration was significantly shorter in medicated and unmedicated patients with chronic schizophrenia at $120 \%$ AMT. ${ }^{96}$ A similar finding was observed in medicated patients with chronic schizophrenia at $110 \%, 130 \%$ and $140 \%$ RMT, but not at $120 \%$ RMT $^{84}$ According to the authors of the latter study, ${ }^{84}$ these findings suggest a deficit in cortical inhibition in patients with schizophrenia that is not medicationrelated, because changes in CSP were not related to drug dose or treatment duration.

Table 2: Overview of results from TMS-EMG studies*

\begin{tabular}{|c|c|c|}
\hline Measure & Main findings & Hypothesis \\
\hline Resting motor threshold & $\begin{array}{l}\text { Fifteen studies reported no significant differences between medicated patients with chronic } \\
\text { schizophrenia and healthy controls; } ; 4,60,78-901 \text { study }{ }^{91} \text { reported no significant difference } \\
\text { between medication-naive or minimally treated ( }<1 \text { month) patients with first-episode } \\
\text { psychosis and healthy controls; } 1 \text { study reported lower RMT in medicated patients; }{ }^{92} \\
2 \text { studies reported higher RMT in medicated patients with chronic schizophrenia; }{ }^{93,94} \\
1 \text { study showed higher RMT in medicated patients }{ }^{45}\end{array}$ & \multirow{2}{*}{$\begin{array}{l}\text { Patients would show deficits in } \\
\text { GABA-mediated cortical inhibition } \\
\text { as measured by CSP and paired- } \\
\text { pulse inhibition paradigms (e.g., } \\
\mathrm{SICl} \text { and } \mathrm{LICl} \text { ). Intracortical } \\
\text { facilitation may not be significantly } \\
\text { different between patients and } \\
\text { healthy controls }\end{array}$} \\
\hline Active motor threshold & $\begin{array}{l}\text { Two studies reported no significant differences between patients with chronic schizophrenia } \\
\text { (mostly medicated) and healthy controls }{ }^{84,86} \text { or healthy siblings }{ }^{86}\end{array}$ & \\
\hline
\end{tabular}

Amplitude of motor evoked potentials

Duration of cortical silent period

Short-latency

afferent inhibition

Transcallosal inhibition

Short-interval intracortical inhibition

Intracortical facilitation

Long-interval intracortical inhibition
Four studies found no significant differences between medicated and unmedicated patients with chronic schizophrenia and healthy controls; ${ }^{81,82,87,94} 2$ studies reported unaltered MEP sizes in medicated patients with first-episode schizophrenia ${ }^{58}$ and medicated patients; 89 1 study found smaller MEP sizes in medicated chronic patients; ${ }^{54} 1$ study found increased MEP sizes in medicated patients with chronic schizophrenia; 881 study found no significant differences between patients with recent-onset and multi-episode schizophrenia, people at risk and healthy controls; 1 study found smaller MEP sizes in people at risk compared to patients with first-episode schizophrenia and healthy controls ${ }^{45}$

Four studies found no significant differences between medicated patients with chronic schizophrenia and healthy controls; ${ }^{45,86,93,95} 2$ studies reported significantly shorter CSP duration in medicated and unmedicated patients with chronic schizophrenia;84,96 1 study found longer CSP duration in medicated patients with chronic schizophrenia and people at risk, and no significant difference between the 2 groups; ${ }^{54} 1$ study reported longer CSP duration in patients with first-episode schizophrenia compared to people at risk and healthy controls, and no difference between the latter 2 groups; 581 study reported longer CSP duration in patients for the $130 \%$ of the RMT but not for $90 \%$ maximum stimulator output ${ }^{94}$

One study reported normal short-latency afferent inhibition in patients compared to healthy controls ${ }^{97}$

Two studies reported increased duration of transcallosal inhibition in patients who used the single-pulse paradigm. ${ }^{79,80}$

Eight studies reported reduced $\mathrm{SICI}$ in medicated patients with chronic schizophrenia, ${ }^{45,54,60,81,82,89,93}$ patients with recent-onset schizophrenia ${ }^{60}$ and medicated patients with first-episode schizophrenia; ${ }^{58} 1$ study reported unchanged $\mathrm{SICl}$ in patients with chronic schizophrenia and medication resistance,95 1 study reported unchanged $\mathrm{SICl}$ in medicated patients with chronic schizophrenia at interstimulus intervals of 1, 2, 3 and $4 \mathrm{~ms}$ but when data for the 4 interstimulus intervals were pooled, patients showed reduced $\mathrm{SICl} ; 84$ 1 study reported reduced $\mathrm{SICI}$ in people at risk; ${ }^{56} 1$ study found unchanged $\mathrm{SICI}$ in people at risk. ${ }^{54}$

Seven studies reported no significant differences between medicated patients with chronic schizophrenia and healthy controls; $45,54,60,82,84,93,951$ study found no significant difference between people at risk, medicated patients with chronic schizophrenia and healthy controls; ${ }^{54} 1$ study reported mixed results: reduced intracortical facilitation in medicated patients with chronic schizophrenia compared to healthy controls in 1 experimental session, but no difference between the groups in another session that was 4 8 days apart ${ }^{89}$

One study found no differences between medicated patients and healthy controls ${ }^{93}$

$\mathrm{CSP}=$ cortical silent period; EMG = electromyography; GABA = $\gamma$-aminobutyric acid; $\mathrm{LICl}=$ long-interval intracortical inhibition; MEP = motor evoked potential; RMT = resting motor

threshold; $\mathrm{SICl}=$ short=interval intracortical inhibition; TMS = transcranial magnetic stimulation.

*Unless otherwise specified, comparisons were made between patient groups or people at risk of psychosis and healthy controls. 
In contrast, Tang and colleagues ${ }^{54}$ found longer CSP durations in medicated inpatients with chronic schizophrenia at $120 \%$ RMT. Longer CSP duration was also found in patients with first-episode schizophrenia compared to healthy controls and people at risk of psychosis using $120 \%$ RMT. ${ }^{58}$ These CSP results suggest that alteration in GABA-B receptor-mediated cortical inhibition could occur during disease progression.

Tang and colleagues ${ }^{54}$ found prolonged CSP duration in people at ultra-high risk of psychosis compared to healthy controls, but no significant difference between medicated patients with chronic schizophrenia and people at ultra-high risk of psychosis. In addition, Soubasi and colleagues ${ }^{94}$ measured CSP using 2 stimulation intensities: 130\% of RMT and $90 \%$ of the maximal stimulator output. They found longer CSP durations in patients than in healthy controls in both hemispheres for $130 \%$ RMT, but not for $90 \%$ of maximal stimulator output. However, they found no group differences when the CSP was expressed as a ratio over the corresponding stimulus intensity (i.e., CSP divided by $130 \%$ RMT) in each participant.

One study applied biphasic TMS pulses over both motor cortices with an intensity of $60 \%$ to $80 \%$ of maximal stimulator output (above the RMT). ${ }^{100}$ The responses were recorded from the abductor digiti minimi muscles in the upper extremities and tibialis anterior muscles in the lower extremities, using needle electrodes inserted into the muscles. The authors observed multiple CSPs in single trials, predominantly in patients with chronic schizophrenia who had been hospitalized and on long-term antipsychotic treatment (averages of 22.5 and 11 years for those on conventional and atypical antipsychotics, respectively). During dominant hemisphere stimulation, the authors found no difference between patients and healthy controls in the latency or duration of the first CSP or in total CSP duration (durations of the first and later CSPs added together for respective stimulation sites); however, patients had a higher number of CSPs in the abductor digiti minimi muscle. Responses from the nondominant hemisphere were similar, except that patients had a longer total CSP duration than healthy controls in the abductor digiti minimi muscle but not a higher number of CSPs. The authors found no significant side-to-side difference in patients or controls for any of the outcome measures. They wrote that multiple CSPs in the upper extremities and in the dominant body side in medicated patients with chronic schizophrenia may suggest impaired regulation of excitatory or inhibitory neurotransmitter systems in the central motor pathway.

The data appear to be highly heterogeneous with respect to the duration of CSP: similar numbers of studies reported increased CSPs, decreased CSPs and no significant change relative to healthy controls. It is possible that disease progression or duration may affect CSP duration.

\section{Short-latency afferent inhibition}

TMS over the primary motor cortex can be paired with stimulation of the peripheral muscle by applying a conditioning afferent stimulus to the medial or ulnar nerve. This protocol, called short-latency afferent inhibition (SAI), elicits MEP inhibition and reflects sensory and motor components.
Pharmacological studies have indicated that SAI may reflect the activity of cholinergic neurotransmission and non- $\alpha 2 / 3$ GABA-A receptors. ${ }^{19,101}$

Differences in SAI between patients and healthy controls were investigated by Noda and colleagues. ${ }^{97}$ They showed that SAI significantly attenuated the size of the MEP evoked by single test pulses in both patients and healthy controls, but they found no difference in the degree of inhibition between groups.

\section{Short-interval intracortical inhibition}

Because SICI reflects the activity of intracortical inhibitory circuits, ${ }^{102,103}$ SICI measurements provide an index for the GABA-A receptor-mediated inhibition of the motor cortex.

In most of the studies we assessed, SICI was significantly reduced in medicated patients compared to healthy controls. ${ }^{45,54,60,81,82,89,93}$ Also compared to healthy controls, SICI was unchanged in patients with chronic schizophrenia and documented medication resistance ${ }^{95}$ and in medicated patients with chronic schizophrenia at interstimulus intervals of 1, 2, 3 and $4 \mathrm{~ms}^{84}$ However, when data for the 4 interstimulus intervals were pooled, the authors observed a significant reduction in the degree of SICI in the patient group. Further, Du and colleagues ${ }^{81}$ showed that smoking status did not affect SICI in patients or controls.

Taken together, these findings of a reduction in SICI suggest a specific deficit in GABA-A receptor-mediated inhibition pathway in this clinical population. Reduced SICI has also been found in patients with recent-onset schizophrenia whose duration of illness was less than 3 years (most were medicated) ${ }^{60}$ and in participants at risk (antipsychotic-naive) and medicated patients with first-episode psychosis and no more than 6 weeks of continuous treatment, ${ }^{58}$ showing that alteration in GABA-A receptor-mediated inhibition may appear early in the disease course. However, 1 study showed unchanged SICI between people at ultra-high risk of psychosis and healthy controls, suggesting that further studies are needed to clarify the timing of any differences in GABA-A receptor-mediated inhibition during the prodromal stage. ${ }^{54}$

\section{Intracortical facilitation}

Most studies reported no significant difference in ICF (indexing the degree of excitation) between medicated patients with schizophrenia and healthy controls. ${ }^{54,82,84,93,95}$ Consistently, 2 other studies reported no differences in ICF between patients (most medicated) and healthy controls. ${ }^{45,60}$ As well, Tang and colleagues ${ }^{54}$ measured ICF and found no significant differences among medicated patients with chronic schizophrenia, drug-naive participants at ultra-high risk of psychosis and healthy controls.

However, Strube and colleagues ${ }^{89}$ investigated ICF before and after the delivery of plasticity-inducing paradigms (paired associative stimulation and anodal tDCS) in separate sessions of approximately 4 to 8 days apart. Compared to healthy controls, patients with chronic schizophrenia (1 unmedicated, 19 medicated) showed reduced $9 \mathrm{~ms}$ ICF and mean 9 to 12 ms ICF values before the delivery of the paired associative stimulation protocol, but no differences between groups before tDCS. 


\section{Long-interval intracortical inhibition}

Only 1 study investigated differences in LICI between medicated patients and healthy controls; results showed no differences in LICI for interstimulus intervals of 100, 150 or $200 \mathrm{~ms}^{93}$

\section{Connectivity measured with TMS-EMG}

TMS-EMG can also be used to investigate connectivity between the 2 motor cortices using a twin-coil paired-pulse paradigm (i.e., transcallosal inhibition), which involves the application of a conditioning stimulus over the motor region, followed by a test stimulus in the homologous contralateral area.

Bajbouj and colleagues ${ }^{79}$ and Boroojerdi and colleagues ${ }^{80}$ used a single-pulse transcallosal inhibition (also called ipsilateral silent period) paradigm, which involves applying single-pulse stimuli over the motor cortex ipsilateral to the contracted hand muscle, to study the connection between the motor cortices of the 2 hemispheres. Both studies reported prolonged duration (measured in milliseconds) of transcallosal inhibition in patients relative to healthy controls. Moreover, Boroojerdi and colleagues ${ }^{80}$ reported increased transcallosal conduction time in medicated patients with chronic schizophrenia, whereas Bajbouj and colleagues $^{79}$ found no difference in the onset latency of transcallosal inhibition between patients with chronic schizophrenia (most of whom were medicated) and healthy controls. In both studies, data recorded from the muscles of both hands were pooled because no side-to-side differences in outcome measures were detected..$^{79,80}$ According to the authors, these findings suggest an abnormal activation of interhemispheric connections between the motor cortices, which may indicate impaired functioning of the corpus callosum in these patients.

Koch and colleagues ${ }^{104}$ also investigated whether schizophrenia affected parietomotor connectivity in the right hemisphere in age-matched patients and healthy controls. In the healthy controls, conditioning stimuli applied over the right posterior parietal cortex with an intensity of 90\% RMT increased the MEP amplitudes elicited by test stimuli applied over the right motor cortex. In comparison, the medicated and unmedicated patient groups showed significantly weaker MEP facilitation when the conditioning stimulus preceded the test stimulus by 2, 4 and $15 \mathrm{~ms}$, suggesting that patients with schizophrenia may have reduced right parietomotor facilitatory connection.

\section{TMS-EEG in schizophrenia}

The outcome measures for the TMS-EEG protocols are described in Table 3. For an overview of results from the TMSEEG studies, see Table 4.

\section{TMS-elicited EEG oscillations in schizophrenia}

\section{TMS-EEG over the primary motor cortex}

Three studies investigated the effect of the paired-pulse paradigm LICI on TMS oscillations over the left primary motor cortex. Farzan and colleagues ${ }^{105}$ compared the TMS-evoked oscillations measured from the C3 electrode between healthy controls and patients with schizophrenia (most of whom were medicated) or bipolar disorder. The authors found no difference in degree of inhibition in the area under the curve for the $\delta, \theta, \alpha, \beta$ or $\gamma$ frequency bands of the TMS-evoked oscillations after LICI and test stimulus alone. They also found no difference in the evoked oscillations in response to test stimulus alone across the groups.

In a later study, Radhu and colleagues ${ }^{99}$ assessed a group of medicated patients with chronic schizophrenia, patients with obsessive-compulsive disorder and healthy controls, analyzing the degree of inhibition across all EEG channels and in the primary motor cortex region of interest by comparing event-related spectral perturbation (ERSP) for the test stimulus alone and for the paired-pulse conditions. The results showed that all groups had significant within-group inhibition after paired-pulses, but no difference in the degree of LICI for any of the frequency bands $(\delta, \theta, \alpha, \beta$ or $\gamma)$. The same authors then used the same method ${ }^{106}$ to analyze the degree of LICI in a region of interest composed of 9 frontal,

Table 3: TMS-EEG outcome measures

\begin{tabular}{|c|c|}
\hline Measure & Description \\
\hline Global mean field power & $\begin{array}{l}\text { Reflects global brain activation, calculated as the root mean-squared value of the signal across } \\
\text { all electrodes }\end{array}$ \\
\hline $\begin{array}{l}\text { Event-related spectral } \\
\text { perturbation }\end{array}$ & $\begin{array}{l}\text { The modulation of amplitude elicited by a specific event (e.g., TMS pulse) relative to a baseline } \\
\text { (e.g., pre-stimulus). The power spectra values of the EEG recorded after TMS are computed for } \\
\text { each trial. Single-trial values are then averaged across all trials and the average baseline power } \\
\text { is subtracted from the whole trial. These are conducted for the relevant EEG channels in the } \\
\text { frequency bands of interest. Event-related spectral perturbation values are independent of the } \\
\text { phase of the evoked or induced activity }\end{array}$ \\
\hline Intertrial coherence & $\begin{array}{l}\text { A measure of phase-locking of the TMS-evoked oscillations across trials; it is independent of } \\
\text { signal amplitude. This measure reflects the degree to which a stimulus induces phase } \\
\text { synchrony (or resetting) of ongoing oscillations at specific frequencies. Intertrial coherence } \\
\text { values range from } 0 \text { (no intertrial coherence) to } 1 \text { (perfect phase alignment across trials) }\end{array}$ \\
\hline
\end{tabular}


frontocentral and central EEG electrodes in medicated patients with chronic schizophrenia, unaffected first-degree relatives of patients and healthy controls, and they found no significant difference in the overall $(2-50 \mathrm{~Hz})$ power spectrum or the $\gamma(30-50 \mathrm{~Hz})$ frequency band among the groups (the data included 6 patients and 6 healthy controls from their earlier study ${ }^{99}$ ).

Ferrarelli and colleagues ${ }^{91}$ stimulated the left primary motor cortex of patients with first-episode psychosis using suprathreshold single-pulse stimuli. Outcome measures included global mean field power, ERSP and intertrial coherence (ITC). Outcomes were calculated across all electrodes and did not differ between patients and healthy controls. However, patients showed significantly decreased relative spectral power in the $27-33 \mathrm{~Hz}$ range in a cluster of frontocentral electrodes that overlay the primary motor cortex. Moreover, the authors reported no difference in any of the main TMS-evoked parameters (global mean field power, ERSP or ITC) between patients with first-episode psychosis who were diagnosed with schizophrenia at 6-month followup and those who were not diagnosed with schizophrenia at follow-up. These findings, showing $\beta$ or low $\gamma$ reduction in first-episode psychosis, indicated that a deficit in TMS-EEG responses may be present at the onset of the illness and was unlikely to be affected by medications, because the patients were either antipsychotic-naive or had limited antipsychotic exposure ( $<1$ month).

\section{TMS-EEG over the premotor cortex}

The combination of TMS-EEG with neuronavigation allows researchers to investigate nonmotor brain regions in schizophrenia, such as the premotor cortex and the dorsolateral prefrontal cortex (dlPFC). ${ }^{6}$ Two studies investigated TMSinduced oscillations by applying single-pulse TMS over the premotor cortex. In both studies, all or most of the patients were medicated; no information was provided about overall symptom severity.

Ferrarelli and colleagues ${ }^{91}$ found $\gamma$-band abnormalities in patients relative to healthy controls between 12 and $100 \mathrm{~ms}$ after TMS. They also examined the ERSP and ITC to better understand whether the $\gamma$ deficit was related to a reduction in amplitude or in a synchronization of the TMS response across trials. They found that both ERSP $\gamma$ values (measuring $\gamma$ amplitude after TMS regardless of the phase) and ITC $\gamma$ values (measuring $\gamma$ synchronization regardless of signal amplitude) were significantly reduced in patients within the first $100 \mathrm{~ms}$.

Table 4: Overview of results from TMS-EEG studies

\begin{tabular}{|c|c|c|c|}
\hline \multirow[b]{2}{*}{ Stimulation site } & \multicolumn{2}{|c|}{ Main findings } & \multirow[b]{2}{*}{ Hypothesis } \\
\hline & Frequency domain & Time domain & \\
\hline $\begin{array}{l}\text { Left primary } \\
\text { motor cortex }\end{array}$ & $\begin{array}{l}\text { Three studies demonstrated no significant difference } \\
\text { in the degree of LICl-induced inhibition of oscillatory } \\
\text { activity in the primary motor cortex }{ }^{99,105} \text { or frontal } \\
\text { region }{ }^{106} \text { between medicated (or medicated chronic) } \\
\text { patients and healthy controls. As well, medicated } \\
\text { patients }{ }^{105} \text { and patients with a first episode of } \\
\text { psychosis }{ }^{91} \text { did not show a significant difference from } \\
\text { healthy controls after single-pulse stimulation of the } \\
\text { left primary motor cortex }\end{array}$ & $\begin{array}{l}\text { Median nerve stimulation (i.e., short- } \\
\text { latency afferent inhibition) induced a larger } \\
\text { increase of the P } 180 \text { amplitude in } \\
\text { medicated patients relative to healthy } \\
\text { controls in the left primary motor cortex }{ }^{97}\end{array}$ & \multirow{3}{*}{$\begin{array}{l}\text { Impaired cortical inhibition and } \\
\text { excitation (mediated by e.g., } \\
\text { GABAergic and glutamatergic } \\
\text { neurotransmission, respectively) in } \\
\text { the frontal lobes of patients would } \\
\text { lead to impairment in the } \\
\text { generation and modulation of TMS- } \\
\text { EEG activity in both the frequency } \\
\text { and time domains. As well, } \\
\text { dysregulation of the central } \\
\text { cholinergic system in patients with } \\
\text { schizophrenia would affect the } \\
\text { modulation of the N100 component } \\
\text { by the SAI paradigm, a marker of } \\
\text { cholinergic activity97 }\end{array}$} \\
\hline Premotor cortex & $\begin{array}{l}\text { One study showed significantly decreased ERSP and } \\
\text { intertrial coherence values in the } \gamma \text { range in } \\
\text { medicated patients within the first } 100 \text { ms post-TMS } \\
\text { in a frontocentral region that included premotor and } \\
\text { sensorimotor areas. }{ }^{83} \text { Another study showed reduced } \\
\text { ERSP in the } \beta / \gamma \text { range and slowed natural frequency } \\
\text { at the stimulation site in medicated patients with } \\
\text { chronic schizophrenia, patients with bipolar disorder } \\
\text { and patients with major depressive disorder } \\
\text { compared to healthy controls }{ }^{107}\end{array}$ & No studies & \\
\hline Left dIPFC & $\begin{array}{l}\text { Relative to healthy controls, chronic (or medicated } \\
\text { chronic) patients with schizophrenia exhibited } \\
\text { reduced } \mathrm{LICl} \text { of } \gamma \text { oscillations in } 2 \text { studies }{ }^{105,106} \text { and of } \\
\text { oscillations in all frequency bands in } 1 \text { study, }^{99} \\
\text { measured by area under the curve }{ }^{105} \text { or ERSP }{ }^{99} \text { in } \\
\text { the dIPFC or by ERSP in a frontal region. }{ }^{106} \text { Two of } \\
\text { these studies also showed reduced } \mathrm{LICI} \text { of } \gamma \\
\text { oscillations in the dIPFC compared to patients with } \\
\text { bipolar disorder, }{ }^{105} \text { and of } \theta, \alpha \text { and } \beta \text { oscillations } \\
\text { compared to patients with obsessive-compulsive } \\
\text { disorder. }{ }^{99} \text { One study demonstrated less SICI- } \\
\text { induced inhibition of evoked } \delta \text { power, and less SICI- } \\
\text { induced inhibition and ICF-induced facilitation of } \\
\text { ERSP in the dIPFC of medicated patients with } \\
\text { chronic schizophrenia relative to healthy controls }{ }^{108}\end{array}$ & $\begin{array}{l}\mathrm{SICI} \text { and ICF induced significantly greater } \\
\text { changes in the amplitude of TMS-evoked } \\
\text { EEG potentials in the left dIPFC in healthy } \\
\text { controls compared to medicated patients } \\
\text { with chronic schizophrenia. }{ }^{108} \mathrm{SAI} \\
\text { increased the N100 amplitude in healthy } \\
\text { controls but not in medicated patients in } \\
\text { the dIPFC. }{ }^{97} \text { Because modulation of N100 } \\
\text { amplitude is a robust primary marker for } \\
\text { SAI, the authors suggested that SAI is } \\
\text { reduced in the dIPFC in patients with } \\
\text { schizophrenia }{ }^{97}\end{array}$ & \\
\hline
\end{tabular}

dIPFC = dorsolateral prefrontal cortex; EEG = electroencephalography; ERSP = event-related spectral perturbation; GABA = $\gamma$-aminobutyric acid; ICF = intracortical facilitation; LICI = long-interval intracortical inhibition; $\mathrm{N} 100=$ negative deflection at around $100 \mathrm{~ms} ; \mathrm{P} 180=$ positive deflection at around $180 \mathrm{~ms}$; SAI = short-latency afferent inhibition; SICI = short-interval intracortical inhibition; TMS = transcranial magnetic stimulation. 
The authors concluded that patients with schizophrenia had an impairment in both the amplitude and phase locking of TMS-induced $\gamma$ responses.

Canali and colleagues ${ }^{107}$ measured ERSP and natural frequency in medicated patients with chronic schizophrenia, bipolar disorder, major depressive disorder and healthy controls over the EEG channel closest to the stimulation site. TMS significantly increased responses on the $\beta-\gamma$ band (range $21 \sim 50 \mathrm{~Hz}$ ) in healthy controls at 20 300 ms after stimulus, whereas the induced or evoked power was significantly lower in patients with bipolar disorder, major depressive disorder or schizophrenia (range 11 27 Hz). These results were in agreement with those of Ferrarelli and colleagues, ${ }^{83}$ who observed reduced ERSP in the $\gamma$ band. Furthermore, frontal natural frequency was significantly slowed in the patient groups compared to healthy controls but did not differ among the patient groups themselves. ${ }^{107}$ According to the authors, impaired frontal natural frequency suggests a common neurobiological mechanism in the pathophysiology of bipolar disorder, major depressive disorder and schizophrenia, which likely involves dysfunction of GABAergic circuits. ${ }^{107}$

These findings showed disrupted $\gamma$ oscillatory activity in patients with schizophrenia and were in line with evidence reporting deficits in $\gamma$ modulation in this population during the administration of cognitive tasks ${ }^{109}$ and perceptive stimuli. ${ }^{110,111}$

\section{TMS-EEG over the dorsolateral prefrontal cortex}

Three studies applied the LICI paradigm over the left dlPFC and found significantly impaired inhibition in patients with schizophrenia: LICI applied over the dlPFC resulted in suppression of the mean cortical evoked activity and also correlated with $\mathrm{LICI}$ in the motor cortex. ${ }^{112}$

Farzan and colleagues ${ }^{105}$ investigated the effect of GABA-B receptor-mediated inhibition on oscillations by applying LICI over the dlPFC. They aimed to explore whether patients with chronic schizophrenia (2 unmedicated, 12 medicated) would show a specific deficit in the ability to inhibit $\gamma$ oscillations compared to healthy controls and patients with bipolar disorder. The authors reported that patients with schizophrenia had a significantly lower degree of LICI (i.e., lower inhibition) in the $\gamma$ band compared to patients with bipolar disorder and healthy controls when EEG activity was measured from the anterior frontal electrode (AF3). They found no differences in other frequency bands.

Radhu and colleagues ${ }^{106}$ found similar results when they investigated LICI in patients, unaffected first-degree relatives and healthy controls. They analyzed data for a region of interest composed of 9 frontal, frontocentral and central electrodes and compared the ERSP between paired-pulse and single-pulse conditions. Healthy controls showed greater LICI-induced inhibition of $\gamma(30-50 \mathrm{~Hz})$ power compared to patients, but there was no difference between healthy controls and unaffected first-degree relatives, or between unaffected first-degree relatives and patients. Furthermore, healthy controls and unaffected first-degree relatives showed greater overall LICI-induced inhibition $(2-50 \mathrm{~Hz})$ compared to patients. Radhu and colleagues ${ }^{99}$ also examined frontal GABA-mediated cortical inhibition in patients with chronic schizophrenia, patients with obsessivecompulsive disorder and healthy controls, testing the hypothesis that frontal inhibition would be reduced in patients with chronic schizophrenia, but not in patients with obsessivecompulsive disorder. They found that when LICI was measured in a dlPFC region of interest using ERSP as the outcome, it was larger in healthy controls than in patients with schizophrenia for all frequency bands, and larger in patients with obsessive-compulsive disorder than in patients with schizophrenia for the $\theta, \alpha$ and $\beta$ bands. They found no significant difference between healthy controls and patients with obsessive-compulsive disorder, and they suggested that these findings provided evidence that the LICI deficit in the dlPFC may be specific to schizophrenia and have potential diagnostic value.

Only 1 study examined whether SICI and ICF of the left dIPFC differentially modulated response to single pulses in medicated patients with chronic schizophrenia and healthy controls. ${ }^{108}$ Analyses showed that SICI induced less inhibitory modulation (i.e., the ratio of conditioned over unconditioned evoked power was greater) in the dlPFC of patients on the $\delta$ band, but with ICF there was no difference between groups in modulation of any frequency band. The time-frequency representations (i.e., ERSPs) of conditioned minus unconditioned power also showed more inhibition and facilitation in healthy controls during SICI and ICF, respectively, suggesting that they had more robust inhibitory and excitatory responses than patients with schizophrenia.

\section{TMS-evoked EEG potentials}

We found only 2 studies that investigated TMS-evoked EEG potentials by applying TMS over the left dIPFC and the primary motor cortex. Noda and colleagues ${ }^{108}$ examined how SICI and ICF of the left dIPFC modulated responses to a single test stimulus in medicated patients with chronic schizophrenia and healthy controls. They found that the decrease in P60 amplitude by SICI was significantly smaller in patients than in healthy controls. In contrast, ICF significantly increased the amplitude of P60 and decreased the amplitude of N100 (i.e., made N100 more positive) in healthy controls but induced no amplitude changes in patients. The topography of amplitude modulation showed that in healthy controls SICI reduced excitation over the left frontal area on P60, but ICF increased excitation on P60 and reduced inhibition on N100 in the left frontal area; these topographical changes were not evident in patients with schizophrenia.

Noda and colleagues ${ }^{97}$ also compared the modulation of TMS-evoked EEG potentials by median nerve stimulation (i.e., SAI) in medicated patients and healthy controls, applying TMS to the left primary motor cortex and the dlPFC. Results showed a significant difference in modulation of N100 in the dlPFC: SAI attenuated the amplitude of N100 in the dlPFC in patients compared to healthy controls. Patients also showed greater modulation of P180 amplitude for SAI in the primary motor cortex. The authors concluded that, although the underlying mechanism for P180 is not yet fully understood, the data indicated altered SAI in schizophrenia, which may reflect altered central cholinergic tone. 


\section{Signal propagation in schizophrenia}

A few studies stimulated the cerebral cortex with TMS and used concurrent EEG to record the spread of neural activity across the brain in the sensor or source space. Ferrarelli and colleagues ${ }^{113}$ stimulated the right premotor cortex of a group of patients with chronic schizophrenia (most of whom were medicated) and healthy controls. They performed source localization analysis for the peaks of global mean field power and showed that within the first $100 \mathrm{~ms}$ after TMS, the centre of neural activity in healthy controls shifted from the premotor cortex to the right sensorimotor areas and then to the left premotor and sensorimotor areas. In patients, cortical activation was more localized, shifting between the premotor and motor areas along the midline in both hemispheres.

Frantseva and colleagues ${ }^{85}$ stimulated the left primary motor cortex with a suprathreshold stimulus intensity in medicated patients with chronic schizophrenia and healthy controls. The patient group showed a higher average global voltage than healthy controls, at around $200 \mathrm{~ms}$ and between $400 \mathrm{~ms}$ and $750 \mathrm{~ms}$ after stimulus, but there was no group difference between $75 \mathrm{~ms}$ and $150 \mathrm{~ms}$ (EEG data analysis started from $75 \mathrm{~ms}$ after stimulus). The voltage distribution topography map suggested that patients experienced more prolonged and widespread activation in response to TMS, hence the higher average global voltage during those time intervals. In the frequency domain, patients showed significantly increased power between $400 \mathrm{~ms}$ and $800 \mathrm{~ms}$ in the $\delta$ band in the left frontal and temporoparietal leads and bilateral occipital and parietal electrodes, and in the $\beta-\gamma$ band in the left central and parietal and right frontal, temporal and central channels.

\section{Plasticity in schizophrenia}

Dysfunctional brain plasticity is a key feature in the pathophysiology of schizophrenia (Haracz $\left.{ }^{114}\right)$. The application of a small ( 1 mA) current across the scalp with tDCS can induce plasticity for up to 1 hour after stimulation. ${ }^{115}$ Typically, anodal tDCS is a facilitatory protocol that increases cortical excitability, whereas cathodal tDCS provokes a suppression. An alternative approach to exploring plasticity is to apply a paired associative stimulation, which induces focal and synapse-specific plasticity at the target neurons, unlike tDCS, which exerts a nonfocal effect. ${ }^{49,50,52,115-118}$

Strube and colleagues ${ }^{89}$ used paired associative stimulation and anodal tDCS to explore differential plasticity response patterns in patients with schizophrenia (19 medicated, 1 unmedicated) and healthy controls. The aim of this study was to investigate whether schizophrenia has a selective impact on focal (induced by paired associative stimulation) versus nonfocal (induced by tDCS) long-term potentiation in the motor cortex. They found that anodal tDCS significantly increased the MEP size in both patients and healthy controls, indicating the successful induction of nonfocal long-term potentiation-like plasticity. In contrast, paired associative stimulation significantly increased the MEP size in healthy controls only, underlining the impairment of focal long-term potentiation-like plasticity in patients with schizophrenia.
SICI and ICF were also measured in this study, before and 15 minutes after the plasticity paradigms, and results showed that anodal tDCS significantly reduced SICI in patients (interstimulus interval $3 \mathrm{~ms}$ ) and increased ICF for interstimulus intervals of 7,9 and $12 \mathrm{~ms}$, but induced no changes in healthy controls. In contrast, paired associative stimulation significantly increased ICF for interstimulus intervals of 7 and $12 \mathrm{~ms}$ in healthy controls, but led to no change in patients. ${ }^{89}$

Hasan and colleagues ${ }^{45}$ also investigated nonfocal longterm potentiation-like plasticity in patients with schizophrenia, paying special attention to the duration and severity of psychosis in recent-onset (single-episode) schizophrenia versus multi-episode schizophrenia. Specifically, they applied anodal tDCS to explore whether patients with schizophrenia would show altered long-term potentiation-like plasticity compared to healthy participants and how these alterations would be related to the course of disease. They also applied different TMS protocols (SICI, ICF, CSP) to determine the physiologic basis of plasticity alterations. Their data showed that anodal tDCS significantly increased the MEP amplitude in both patient groups and healthy controls. However, patients with multi-episode (but not recent-onset) schizophrenia showed less MEP facilitation compared to controls. Furthermore, anodal tDCS enhanced SICI (i.e., induced more inhibition) in the recent-onset group only and changed the CSP duration in all 3 groups, although the direction of change was not specified. The authors reported no difference for ICF. Because of a significant difference in age between controls and patients with multi-episode schizophrenia, they conducted a subanalysis in which the 2 groups were matched for age, sex, handedness and smoking status $(n=10$ in each group) and found greater MEP facilitation in healthy controls compared to patients with multi-episode schizophrenia after anodal tDCS; no other parameters differed among groups. According to the authors, these results indicated that longterm potentiation-like plasticity is related to disease course, and deficits may be because of disturbances in NMDA, GABA and dopamine receptors. ${ }^{45}$

Fitzgerald and colleagues ${ }^{96}$ also found reduced plasticity responses in patients with schizophrenia in a study investigating the cortical response to a 15 minute train of $1 \mathrm{~Hz}$ repetitive TMS (rTMS) on measures of cortical excitability (RMT, AMT and MEP) and inhibition (CSP). Results showed that rTMS significantly increased RMT and AMT in healthy controls but not in medicated or unmedicated patients with schizophrenia. As well, the stimulation protocol induced an increase in MEP amplitude in both patients and healthy controls, but it reduced the CSP duration in healthy controls only. The medicated and unmedicated patient groups did not differ in their response to rTMS based on any measure. Moreover, when data for both patients and controls were pooled, the authors found that a longer CSP duration at baseline was associated with stronger changes in RMT after rTMS. ${ }^{96}$ These results support the hypothesis that deficits in cortical inhibition in patients with schizophrenia may drive reduced brain response to rTMS.

Neural plasticity can be evaluated using a paradigm called use-dependent plasticity, which involves several steps. ${ }^{44}$ First, the spontaneous direction of TMS-induced thumb movements 
is observed; second, patients are trained to move their thumbs in the opposite direction for 30 minutes; finally, after training, the TMS-induced thumb movements are measured again. The authors measured motor plasticity, evaluating the direction and acceleration of thumb movements. Medicated $(n=14)$ and unmedicated $(n=6)$ patients showed an equal deficit in orienting post-training thumb movements in the direction of training compared to healthy controls. The authors found no significant difference in TMS-induced amplitude of thumb movements before or after training across groups, suggesting that the excitability of the motor cortex did not differ. However, the failure of post-training movements to orient in the direction of training suggests that neurophysiological mechanisms involved in such reorientation are disrupted in schizophrenia. Since both NMDA and GABA receptor-mediated neurotransmission have been shown to have important effects on such use-dependent plasticity, ${ }^{44}$ the observed neural plasticity deficit in schizophrenia may be related to these neurotransmitters.

\section{Effects of medication}

Antipsychotic medications are the main treatment for psychosis symptoms in several psychotic disorders, including schizophrenia, schizoaffective disorder, delusional disorder and bipolar affective disorder. Treatment may include antipsychotic medication as monotherapy or with adjunct antidepressants, mood stabilizers or benzodiazepines. ${ }^{119}$ All available antipsychotic medications act by reducing dopamine-dependent postsynaptic activity, reflecting the central role of the dopamine neurotransmitter system in the pathophysiology of psychosis symptoms. ${ }^{120}$ However, up to a third of patients treated with antipsychotics fail to show a positive response, suggesting that other mechanisms are at play. Certainly, antipsychotic medications differentially affect the GABAergic and glutamatergic systems in the cortex, suggesting that their effects are not limited to their action on dopamine receptor systems. Olanzapine has been shown to determine downregulation of the GABA-A receptor in rats, but haloperidol and chlorpromazine do not produce the same effects. ${ }^{121}$ Further, olanzapine, risperidone and other antipsychotics appear to have different effects on biobehavioural changes induced by phencyclidine, suggesting that these medications may exert a different action on NMDA receptors. ${ }^{122,123}$ Finally, clozapine, which has been shown to be the only effective antipsychotic for treatment-resistant patients, modulates glutamatergic activity that has been correlated with the degree of symptomatic change, ${ }^{124}$ further supporting the idea of other mechanisms underlying its therapeutic effects. ${ }^{19,125-127}$

It is possible that a more general systems-level explanation may lie in the possibility that antipsychotic medication exerts its effect by altering the cortical E/I balance in patients with schizophrenia. A systematic review of prospective proton magnetic resonance spectroscopy studies indicated an effect of antipsychotics on E/I balance by showing that most of the studies found a reduction in glutamate in the brains of patients with schizophrenia after longitudinal antipsychotic treatment. ${ }^{128}$ As well, deficits in motor cortical inhibition have been linked to schizophrenia symptom severity (as assessed by the Positive and Negative Syndrome Scale). ${ }^{87}$
These findings indicate that antipsychotic medications may exert their therapeutic effect by normalizing E/I balance in the brain. Based on this assumption, we included studies that investigated the relationship between TMSEMG or TMS-EEG parameters and medication dose, as well as differences in these parameters before and after treatment and between medicated patients, unmedicated patients and healthy controls.

\section{TMS-EMG}

Most studies showed that antipsychotic treatment had no effect on RMT, AMT or MEP amplitudes in medicated patients compared to unmedicated patients and healthy controls. ${ }^{96,98,104,129}$ However, Daskalakis and colleagues ${ }^{130}$ showed that, compared to unmedicated patients, treated medicated patients had a higher RMT, similar to values in healthy controls. The authors concluded that membrane excitability may be lower in unmedicated patients than in medicated patients and healthy controls.

Moreover, Ribolsi and colleagues ${ }^{129}$ examined interhemispheric connections between the dorsal premotor cortex and the contralateral primary motor cortex in schizophrenia. Specifically, they examined the activation of distinct facilitatory and inhibitory transcallosal pathways between these 2 regions by adjusting the intensity of dorsal premotor cortex stimulation to be either suprathreshold (110\% of RMT) or subthreshold ( $80 \%$ of AMT). They found that, relative to healthy controls, medicated patients (but not unmedicated patients) showed significantly less MEP amplitude facilitation by the facilitatory conditioning stimulus (applied over the left dorsal premotor cortex) when the conditioning stimulus preceded the test stimulus (applied over the right primary motor cortex) by $8 \mathrm{~ms}$. However, inhibition of MEP amplitudes by the inhibitory conditioning stimulus did not differ across groups. These results suggest that medication reduced interhemispheric facilitation but did not change inhibitory connectivity between the left dorsal premotor cortex and right motor cortex.

The findings for SICI were variable. Daskalakis and colleagues ${ }^{130}$ reported enhanced SICI with medication to the level of healthy controls, whereas Fitzgerald and colleagues ${ }^{96}$ reported reduced SICI with medication compared to healthy controls. Both studies found no statistical difference in SICI between medicated and unmedicated patients and no effect of medications on ICF. Of the 4 studies that compared unmedicated patients to healthy controls, none reported group differences in ICF. ${ }^{87,96,130,131}$ Only Daskalakis and colleagues ${ }^{130}$ found reduced SICI in unmedicated patients; the other 3 studies $^{87,96,131}$ reported no group difference in SICI. The effects of medication on SICI are not yet clear, because 2 of the studies $^{96,130}$ demonstrated that antipsychotic treatment might have changed SICI (although in the opposite direction) relative to healthy controls, but all 4 studies showed no significant difference between medicated and unmedicated patients. ${ }^{87,96,130,131}$ As for ICF, our findings showed no difference among medicated patients, unmedicated patients and healthy controls, suggesting that this parameter is unlikely to be affected by medications. 
Similar results have been reported for LICI and I-wave facilitation. Fitzgerald and colleagues ${ }^{98}$ examined LICI and I-wave facilitation in patients with schizophrenia and found no difference in LICI among medicated and unmedicated patients and healthy controls. At an interstimulus interval of $1.2 \mathrm{~ms}$, the degree of I-wave facilitation was greatest in medicated patients and lowest in healthy controls, with unmedicated patients occupying an intermediate position (not significantly different from the other 2 groups). The authors concluded that antipsychotic medications did not rectify the deficits underlying I-wave facilitation in patients; instead, they exacerbated them.

Two studies that examined the effect of medications on CSP duration also provided inconclusive results. Fitzgerald and colleagues $^{96}$ reported that medications did not change CSP duration: both medicated and unmedicated patients had shorter CSP durations than controls. In contrast, Daskalakis and colleagues ${ }^{130}$ showed increased CSP duration in medicated patients compared to unmedicated patients, and similar CSP duration between medicated patients and healthy controls. Further, Ahlgren-Rimpilainen and colleagues ${ }^{100}$ found that in the nondominant extremities (i.e., the nondominant hemisphere, abductor digiti minimi and tibialis anterior muscles), patients who were taking conventional antipsychotics had shorter mean first and total CSP duration compared to healthy controls and patients who were taking atypical antipsychotics; those taking atypical antipsychotics had the longest mean first and total CSP durations. According to the authors, ${ }^{100}$ these findings were in line with the assumption that clozapine, which is an atypical antipsychotic, may normalize or even lengthen reduced central inhibition in schizophrenia.

Reports have indicated that CSP duration is increased in patients treated with clozapine compared to healthy controls, unmedicated patients and patients treated with other antipsychotics. ${ }^{87,131}$ Further, Kaster and colleagues ${ }^{95}$ also found that 6 weeks of clozapine treatment increased the CSP duration in patients with medication resistance, and Yildiz and colleagues ${ }^{90}$ showed that 8 weeks of clozapine treatment increased the CSP duration in 6 patients with moderate positive symptoms compared to healthy controls. Taken together, these results suggest that the therapeutic effect of clozapine may be in part through GABA-B receptor-mediated inhibition. ${ }^{6}$

CSP duration is also affected by risperidone, another atypical antipsychotic. Ustohal and colleagues ${ }^{132}$ treated 13 hospitalized medication-naive patients (first-episode psychosis) with risperidone for 4 weeks and found that this antipsychotic significantly increased the CSP duration. The authors concluded that these findings indicated an association between risperidone monotherapy and an increase in GABA-B receptor-mediated inhibition.

Clozapine has not been demonstrated to alter RMT, MEP size (in response to suprathreshold single-pulse stimuli), SICI or ICF, $, 7,90,95,131$ although Yildiz and colleagues ${ }^{90}$ reported decreased ICF after 8 weeks of clozapine treatment. Risperidone had no effect on RMT, SICI or ICF after 4 weeks of treatment ${ }^{132}$ or compared to healthy controls. ${ }^{133}$ Similarly, olanzapine did not change RMT, SICI, or ICF compared to healthy controls. ${ }^{133}$
Interestingly, studies that compared patients taking different antipsychotics showed differences in TMS-EMG biometrics related to these medications. Specifically, Fitzgerald and colleagues ${ }^{133}$ investigated differences in cortical inhibition in patients who received treatment with olanzapine and risperidone. Results showed that patients taking risperidone had significantly higher RMT than those taking olanzapine; neither medication group differed significantly from healthy controls.

As well, olanzapine increased the duration of (singlepulse) transcallosal inhibition relative to risperidone and controls; risperidone decreased the duration of (dual-pulse) transcallosal inhibition relative to olanzapine and healthy controls. ${ }^{133}$ Both medication groups showed less reduction in the conditioned MEP size than healthy controls during dual-pulse transcallosal inhibition when the hand muscle was at rest. According to the authors, these findings suggest that olanzapine and risperidone have differential effects on cortical inhibition and on different aspects of cortical excitability, which might include differences in the action of the medications on GABA or glutamate, directly or through modulation of ascending amine systems. Similarly, Soubasi and colleagues ${ }^{94}$ showed that patients receiving olanzapine had lower RMT and the lowest stimulus intensity required to produce the maximum MEP in the hand muscle than those taking ziprasidone when stimulated over the left hemisphere.

Finally, 7 studies examined the relationship between TMSevoked muscular responses and medication dose in patients. ${ }^{58,60,84,89,100,104,129}$ No significant correlation was reported between antipsychotic dose and RMT, AMT, MEP amplitude elicited by single suprathreshold stimuli, CSP duration or left dorsal premotor-right motor facilitatory connectivity. ${ }^{58,84,89,100,104,129}$ Further, the degree of SICI and ICF was not related to the dose of antipsychotics ${ }^{58,84,89}$ or benzodiazepines. ${ }^{60}$

\section{TMS-EEG}

We found only 3 studies that investigated the effect of medications on TMS-EEG parameters. Ferrarelli and colleagues ${ }^{91}$ found no significant difference in TMS-EEG parameters (global mean field power, ERSP, ITC and relative spectral power) between patients with first-episode psychosis who were antipsychotic-naive versus those who were taking antipsychotics. Noda and colleagues ${ }^{97}$ examined the effect of SAI on TMS-evoked EEG potentials between patients treated with clozapine $(n=4)$ and not treated with clozapine $(n=8)$, by stimulating the left primary motor cortex and dIPFC and reported no difference between groups. Radhu and colleagues ${ }^{106}$ also investigated differences in the degree of inhibition induced by LICI (applied to left dIPFC) in patients treated with antidepressants $(n=15)$ or benzodiazepines $(n=15)$. They found that both groups had a similar degree of inhibition in the overall $(2-50 \mathrm{~Hz})$ and $\gamma$ frequency bands.

Furthermore, 6 studies that examined the association between TMS-EEG outcomes and antipsychotic dose showed no relationships between these 2 parameters. ${ }^{83,97,99,105-107}$ Specifically, no association was found between antipsychotic dose and oscillations (i.e., ERSP or ITC in the $\gamma$ band; degree 
of LICI overall and in the $\gamma$ band; natural frequency) or the modulation of the components of TMS-evoked EEG potentials induced by SICI and ICF.

There was an absence of any robust effects of antipsychotic medication on TMS-EEG parameters; further studies using larger samples may be needed to better address how TMSEEG biometrics can reveal differences between drug-naive and medicated patients in terms of brain responses to medications, as well as how medication doses can affect these parameters.

\section{Relationship with clinical ratings in patients with schizophrenia}

Some studies investigated the relationship between TMSEMG and TMS-EEG parameters and symptom severity as assessed by different scales, such as the Positive and Negative Syndrome Scale (PANSS), the Scale for the Assessment of Positive Symptoms (SAPS), the Scale for the Assessment of Negative Symptoms (SANS), the Brief Psychiatric Rating Scale (BPRS), the Global Assessment Scale and the Global Assessment of Functioning scale.

The symptom scales differ from each other in their components and focus. The most widely used is the PANSS (30 items), ${ }^{134}$ an instrument for assessing the prevalence of positive and negative symptoms in schizophrenia using 2 different subscales: a positive symptoms subscale $(7$ items $)$ and a negative symptoms subscale (7 items). The PANSS also includes a third general psychopathology subscale (16 items) that considers the positive-negative syndrome relative to the general severity of psychopathology. The SAPS 135 and SANS ${ }^{136}$ scales are used to assess positive and negative symptoms in schizophrenia, respectively. The SANS scale is composed of 5 domains (active flattering or blunting; alogia; avolition or apathy; anhedonia or asociality; and attention), and the SAPS is composed of 4 domains (hallucinations; delusion; positive formal thought disorder; and bizarre behaviour). For both scales, symptoms are rated from 0 to 5 (absent to severe). In a similar vein, the BPRS ${ }^{137}$ is used for the global psychopathological evaluation of psychiatric symptoms, rated from 1 to 7 , and is composed of 24 items.

Scales for a more global assessment of the patient's function include the Global Assessment Scale, ${ }^{138}$ a tool used to evaluate the overall functioning of a patient during a specified time period on a continuum from psychological or psychiatric sickness to health. Values range from 1 (the hypothetically sickest individual) to 100 (the hypothetically healthiest). Similarly, the Global Assessment of Functioning scale $^{139}$ considers psychological, social and occupational functioning on a hypothetical continuum of mental health to illness and is used to index the severity of psychopathology (values range from 1 to 100).

\section{TMS-EMG}

Most of the studies we assessed found a relationship between symptom severity and MEP size evoked by single suprathreshold stimulus, although the significance of this relationship remains unclear. In 1 study, ${ }^{87}$ this parameter was positively correlated with PANSS total scores and positive, negative and general psychopathology subscale scores, but it had a negative correlation with PANSS positive subscale scores and a positive correlation with PANSS negative subscale scores in another. ${ }^{54}$ One study ${ }^{96}$ reported no relationship between MEP size and PANSS scores in patients with schizophrenia.

The most consistent finding between TMS-EMG parameters and symptom severity is the association between SICI and PANSS scores. Daskalakis and colleagues ${ }^{131}$ reported a positive correlation between SICI and PANSS total and positive subscale scores (i.e., the lower the inhibition, the higher the score) in a group of patients with chronic schizophrenia, most of them medicated. They found the same result ${ }^{130}$ in medicated and unmedicated patients with chronic schizophrenia (pooled). They also reported that reduced inhibition indexed by SICI was correlated with higher scores on the general psychopathology subscale. ${ }^{130}$ In addition, Liu and colleagues ${ }^{87}$ found that when their patient group was split into unmedicated and medicated subgroups, they observed a significant correlation between SICI and PANSS total scores in the unmedicated group, and no correlation for the medicated group. These findings highlight a potential relationship between symptom severity and GABA-A receptor-mediated inhibition deficits in patients with schizophrenia, which may be altered by antipsychotic treatment. ${ }^{87}$ Furthermore, none of these studies found a correlation between SICI and scores on the PANSS negative subscale, and 3 studies reported no correlation between SICI and clinical rating as assessed by the PANSS. ${ }^{60,84,96}$

Only 2 studies reported an association between CSP duration and clinical severity. Shortened CSP was correlated with higher negative symptoms in medicated and unmedicated patients with chronic schizophrenia (pooled). ${ }^{87}$ Tang and colleagues ${ }^{54}$ reported a positive correlation between CSP and the PANSS positive subscale (i.e., longer the CSP duration, the higher the score) in a group of medicated inpatients. Together, these results suggest that the pathophysiology of symptoms might in part be led by GABA-B receptor-mediated cortical inhibition..$^{54,87}$ In contrast, 4 studies ${ }^{82,96,100,130}$ reported no correlation between CSP duration and symptom severity as assessed by the PANSS. Moreover, a change in CSP duration was not related to a change in PANSS scores after 6 weeks of clozapine treatment in a group of treatmentresistant patients, ${ }^{95}$ and an increase in CSP duration was not related to a decrease in PANSS scores after 4 weeks of risperidone treatment. ${ }^{132}$

With respect to ICF, Daskalakis and colleagues ${ }^{131}$ found a positive correlation with the PANSS positive subscale in medicated and unmedicated patients (pooled), but they thought that this finding was because ICF was mediated, in part, by SICI in the cortex. Tang and colleagues ${ }^{54}$ found that a higher negative symptom score was associated with a lower ICF among medicated patients with schizophrenia, but they concluded that this relationship needed further investigation. In another study, ${ }^{90}$ a reduction in ICF (interstimulus interval $=7 \mathrm{~ms}$ ) was associated with a reduction in scores on the PANSS general psychopathology subscale in a group of patients with moderate positive symptoms after 8 weeks of treatment with an atypical antipsychotic. According to the 
authors, this result may have been because of an increase in cortical inhibition after antipsychotic treatment. In contrast to these findings, 3 studies found no relationship between ICF and PANSS scores. ${ }^{84,96,130}$

The few studies that assessed the relationship between neural connectivity and symptom severity had inconclusive results. Ribolsi and colleagues ${ }^{129}$ found that when the conditioning stimulus intensity was $80 \%$ AMT and the interstimulus interval was $8 \mathrm{~ms}$, lower negative symptoms in medicated and unmedicated patients with chronic schizophrenia (pooled) were associated with a less impaired facilitatory connection between the left dorsal premotor cortex and the right primary motor cortex; they found no correlation with the PANSS positive subscale or daily functioning as assessed by the Global Assessment of Functioning scale. Koch and colleagues ${ }^{104}$ found that the amount of facilitatory connection from the right posterior parietal cortex to the right motor cortex (for conditioning stimulus intensity of $90 \%$ RMT) in patients with chronic schizophrenia (14 medicated, 6 unmedicated) was positively correlated with scores on the Global Assessment of Functioning scale and negatively correlated with scores on the PANSS negative subscale, showing that patients with better global functioning and fewer negative symptoms had less impaired connectivity between the 2 regions. In contrast, Bajbouj and colleagues ${ }^{79}$ found no relationship between the onset and duration of single-pulse transcallosal inhibition and symptom severity (PANSS and BPRS scores) or mental health and functioning (Global Assessment Scale score) in patients with chronic schizophrenia (most of them medicated). Similarly, Daskalakis and colleagues ${ }^{130}$ found no correlation between PANSS scores and dual-pulse transcallosal inhibition, measured by the degree of inhibition of the MEP size by conditioning stimuli when the ipsilateral hand muscle was at rest.

In patients with schizophrenia, no correlation was found between RMT and symptom severity or health functioning as measured by the PANSS, the BPRS and the Global Assessment Scale. ${ }^{79,84,96,130}$ Similarly, there was no association between I-wave facilitation and the PANSS ${ }^{98}$ and between AMT and the PANSS.

\section{TMS-EEG}

Only 1 study examined correlations between TMS-evoked EEG potentials and clinical ratings, ${ }^{108}$ patients demonstrated less attenuation of N100 amplitude by ICF on the dlPFC, suggesting a reduced glutamatergic excitatory effect, and they had lower clinical symptom severity as assessed by PANSS total score. ${ }^{108}$

Two studies investigated the relationship between symptom severity and TMS-elicited oscillations induced by stimulating the primary motor cortex, and they reported inconsistent results. Frantseva and colleagues ${ }^{85}$ found that patients with chronic schizophrenia (14 medicated, 2 unmedicated) showed increased TMS-induced oscillations at late time intervals (400-800 ms) after stimulating the primary motor cortex. They found that $\gamma$ power (the maximum power for the time period of interest averaged across 60 electrodes for each participant) between $400 \mathrm{~ms}$ and $700 \mathrm{~ms}$ was positively correl- ated with positive symptoms; negative symptoms were positively correlated with excessive propagation in the $\theta$ and $\delta$ bands at $200 \mathrm{~ms}$. According to the authors, because the excessive spread of $\gamma, \theta$ and $\delta$ activity may be because of a deficit of cortical inhibition in the primary motor cortex, it may be linked to schizophrenia symptomatology. ${ }^{85}$ In contrast, Ferrarelli and colleagues ${ }^{91}$ reported that ERSP and ITC (averaged between 8 45 Hz and 20-300 ms, stimulated left primary motor cortex) were not associated with positive and negative symptoms scores as measured by SAPS and SANS in first-episode psychosis. However, relative spectral power analysis showed that reduced frontal $\beta$ and low $\gamma$ activity (in a cluster of frontocentral electrodes overlying the motor cortex) was related to higher SAPS and SANS scores. Based on these findings, the authors concluded that reduced TMS-elicited frontal fast oscillations may be associated with the severity of symptoms in patients with first-episode psychosis.

Different results were described in a study that explored stimulation of the dlPFC. Radhu and colleagues ${ }^{106}$ found that inhibition of $\gamma$ power after LICI of the left dlPFC was not associated with BPRS scores, and frontal natural frequency after TMS of the left premotor cortex was not correlated with PANSS scores.

These few findings show a potential link between TMSEEG biometrics and symptom severity, but this relationship needs further investigation to better understand the utility of these measures in the evaluation of disease progression.

\section{Relationship with illness duration in patients with schizophrenia}

\section{TMS-EMG}

Only 1 study reported a significant correlation between illness duration and TMS-EMG parameters. Koch and colleagues ${ }^{104}$ found that the mean amount of facilitation across interstimulus intervals (induced by conditioning stimulus with an intensity of $90 \%$ RMT over the right posterior parietal cortex) correlated positively with illness duration in patients with chronic schizophrenia (14 medicated, 6 unmedicated). These results suggest that longer illness duration is associated with a better facilitatory connection between the right posterior parietal and right motor cortices.

Ribolsi and colleagues ${ }^{129}$ showed a lack of relationship between the facilitatory connection for the left dorsal premotor and right motor cortices (stimulation intensity of $80 \%$ AMT and $8 \mathrm{~ms}$ interstimulus interval) and illness duration in a group of patients with schizophrenia (most of whom were under antipsychotic treatment). Furthermore, 3 of the 6 studies we assessed reported no correlations between illness duration and the following: SICI in patients with recent-onset schizophrenia; ${ }^{60}$ RMT, onset latency and duration of singlepulse transcallosal inhibition in patients with chronic schizophrenia (most of them medicated) $;^{79}$ and CSP duration in long-term (> 1 year) medicated patients with chronic schizophrenia. ${ }^{100}$ As well, RMT, SI-1mV MEP size, SICI, ICF and CSP duration were not related to the number of psychotic episodes or duration of psychosis in patients with recentonset or multi-episode psychosis. ${ }^{45}$ 


\section{TMS-EEG}

Only 1 study investigated the relationship between TMSEEG parameters and illness duration in a group of patients with chronic schizophrenia, most of whom were medicated. ${ }^{83}$ Results showed that after stimulation of the right premotor cortex, ERSP and ITC values in the $\gamma$ band were not correlated with illness duration in patients with schizophrenia.

\section{Relationship with cognition in patients with schizophrenia}

As reported in a growing body of literature, abnormalities in cognitive functioning may represent a key component of schizophrenia. ${ }^{140,141}$ Several studies have investigated the associations between impaired cognition as assessed by neuropsychological tests and TMS-EMG or TMS-EEG biometrics in schizophrenia.

The most consistent finding in this field is again related to SICI, supporting the idea that deficits in GABA-A receptormediated inhibition may be associated with cognitive impairment in schizophrenia. Takahashi and colleagues ${ }^{60}$ found that higher inhibition indexed by SICI in patients with recent-onset schizophrenia (duration of illness less than 3 years) was associated with better working memory capacity as measured by the Brief Assessment of Cognition in Schizophrenia (BACS-J). ${ }^{142}$ Similarly, Noda and colleagues ${ }^{108}$ showed that deficient reduction of $\mathrm{P} 60$ amplitude by SICI (over the left dlPFC region of interest) in medicated patients with chronic schizophrenia was associated with worse performance in the Letter-Number Span test. Specifically, longer the letter-number span, the greater the reduction in P60 amplitude.

These results suggest that better working memory capacity may be associated with a higher level of inhibition, indicated by increased SICI. In addition, Bridgman and colleagues ${ }^{93}$ found that when data from patients and healthy controls were pooled, increased SICI was associated with better task accuracy on the 3-back condition of the n-back task, suggesting that better working memory accuracy is associated with more inhibition. The authors suggested that although this relationship was not significant when separated by diagnosis in their study, these results support a linear relationship between GABAergic inhibition and working memory function.

Changes in SICI have also been positively correlated with changes in auditory verbal memory performance (as indicated by the delayed recall test [A7] of the Rey Auditory Verbal Learning Test) and negatively correlated with task switching and response suppression (as indicated by score on the Stroop Colour and Word Test; analyzed the time taken to complete the test) in a group of patients with chronic schizophrenia who were treated with an antipsychotic. ${ }^{90}$

Lindberg and colleagues ${ }^{86}$ applied SICI in patients with chronic schizophrenia (22 medicated, 7 unmedicated), healthy siblings of patients with schizophrenia and healthy controls during a stop signal task. Specifically, they studied task-related changes in motor excitability (response to single test stimulus) and inhibition (response to paired stimuli) using the task, which assesses the ability to inhibit a prepared action. Results showed that task performance was similar among the groups in terms of the time taken to inhibit a prepared movement (i.e., stop signal reaction time). SICI was modulated by the task (i.e., SICI was lowest during preparation of the action and increased during successful inhibition) in healthy controls and siblings. However, SICI was reduced in patients during motor inhibition, despite equivalent task performance. The authors concluded that in schizophrenia, task-related deficiency in SICI modulation may reflect deficient GABA-A-related processing in the motor cortex, and that the absence of differences in motor inhibition performance between patients and controls could be explained by a compensatory activity of premotor areas.

As for LICI, Lett and colleagues ${ }^{143}$ found that the presence of a "risk genotype" (i.e., GAD1 T-allele) predicted a lower degree of inhibition as measured by LICI after left dlPFC stimulation in patients. The authors showed that the GAD1 risk genotype was also a significant predictor of performance on the Letter-Number Sequencing test, the digit span test and the Stroop Colour and Word Test after covarying for age and IQ in a general linear model (because age and IQ correlated with cognitive test scores). These results suggest that the relationship between genetic factors, working memory performance and the Stroop effect could be mediated in part by metabotropic GABA-B inhibitory neurotransmission.

As well, 1 study found a negative correlation between SAI-induced modulation of the N100 amplitude in the left dlPFC and executive function (visual attention and task switching) in patients, ${ }^{97}$ suggesting that greater N100 attenuation by SAI was associated with poorer performance on the Trail-Making Test.

Finally, 4 studies investigated mirror neuron activity in patients with schizophrenia, because abnormal cortical inhibition has been hypothesized to underlie the social cognition deficit in this population, and the mirror neuron system has been proposed as a neurophysiological substrate for social cognition. ${ }^{78,144-146} \mathrm{~A}$ common method for assessing mirror neuron activity is to compare TMS-induced activity during action observation to that during observation of a static image. Mehta and colleagues ${ }^{145}$ used 4 TMS paradigms eliciting a MEP in the first dorsal interosseus] muscle: LICI, SICI, RMT and the minimum stimulation intensity for evoking $1 \mathrm{mV}$ peak-to-peak amplitude. These were applied as participants observed a goal-directed action (i.e., actual action and its video) involving the first dorsal interosseus and a static image. Differences in the amplitude of the MEP and the degree of cortical inhibition while participants observed the static image and the action provided a measure of mirror neuron activity. Healthy controls and medicated patients showed increased MEP amplitudes and reduced SICI compared to the "rest" state (i.e., the static image). In contrast, drug-naive patients showed deficient MEP enhancement during action observation, suggesting a lack of mirror neuron activity, as well as reduced modulation of SICI by mirror neuron activity. The authors also found that mirror neuron activity had significant positive associations with theory of mind (ability to infer intentions, dispositions and beliefs of others), as measured by the Social Cognition Rating Tool in Indian Setting (SOCRATIS) in drug-naive patients and when both patient groups were pooled. ${ }^{145}$ 
Mehta and colleagues ${ }^{144}$ also showed that drug-naive patients had reduced SICI compared to healthy controls when viewing a static image, and this was inversely associated with the ability to process emotions from facial cues (the emotion recognition index) and overall social cognition ability (the social cognition composite score) as measured by SOCRATIS and the Tool for Recognition of Emotions in Neuropsychiatric Disorders. The authors suggested that these results support the concept of associations between mirror neuron activity and social cognition abilities in patients with schizophrenia, and that impaired mirror neuron activity mediation with SICI supports a role for impaired GABA-A neurotransmission underlying deficits in mirror neuron activity in patients with schizophrenia.

Basavaraju and colleagues ${ }^{146}$ also studied mirror neuron activity in patients with chronic schizophrenia, with and without ego-boundary disturbances-a cluster of psychotic symptoms (including delusions of alien control, thought alienation phenomena and somatic passivity) that involves an alienation of the sense of one's own mental or physical activity. Participants (most of whom were medicated) underwent the same rest, actual observation and virtual observation tasks as described in Mehta and colleagues. ${ }^{145}$ Suprathreshold single-pulse stimuli evoked greater MEP amplitudes in patients without ego-boundary disturbances during action observation, indicating less mirror neuron activity in patients with ego-boundary disturbances during action observation. However, the authors found no significant difference in mirror neuron activity between the 2 patient groups using SICI or LICI.

More recently, Bagewadi and colleagues ${ }^{78}$ studied mirror neuron activity during context-based versus neutral action observation in patients and healthy controls. Neutral action observation required participants to watch a video that depicted the experimenter's hand holding a key in a lateral pinch grip and performing repeated locking and unlocking actions; context-based action observation involved a video that depicted the same action but in a context with social and emotional stimuli. The authors found that mirror neuron activity was greater during context-based action observation (contextbased minus rest state) than in neutral action observation (neutral minus rest state) in both groups, shown as higher SI- $1 \mathrm{mV}$ MEP size, less SICI and more ICF, meaning that context-based action observation elicited more excitation than neutral action observation. These results demonstrated that providing a context to the action modulated mirror neuron activity; however, such an increase in mirror neuron activity during contextbased action observation was reduced in patients relative to healthy controls for SI-1mV MEP size and SICI, but not ICF. They also found that mirror neuron activity as measured by the ICF paradigm during context-based action observation was positively correlated with social cognition performance as assessed by comprehensive social cognition assessments.

\section{Risk of bias assessment}

Of the 42 studies we assessed, 34 (80\%) showed a low risk of bias for participant selection, 38 (90\%) showed a low risk of bias for incomplete outcome data and 42 (100\%) showed a low risk of bias for selective reporting. However, $37(88 \%)$ and $36(86 \%)$ studies were considered unclear for measurement of exposure and blinding of outcome assessment, respectively, because even though research methods were described, details about blinding were not available. Further, $23(55 \%)$ showed a high risk of bias for confounding variables because the major confounding variables (e.g., age, sex or any additional factors) were not adequately confirmed and considered during the design or analysis phase (e.g., through matching, participation restriction or other methods). The results for risk of bias assessment for each study are summarized in Appendix 1, Table S3.

\section{Discussion}

\section{TMS for measuring excitatory and inhibitory processes in schizophrenia}

SICI was reduced in patients with schizophrenia in 9 out of 11 studies that measured SICI. SICI is thought to reflect postsynaptic inhibition of corticospinal neurons mediated by $\alpha 2$ or $\alpha 3$ subunit-bearing GABA-A receptors. ${ }^{19,40}$ A recent systematic review suggested that in schizophrenia, expression of GAD67 mRNA is diminished in a subset of parvalbumincontaining GABAergic interneurons in the primary motor cortex and dlPFC, accompanied by a decreased level of type 1 GABA transporter mRNA, which may result in a net increase or decrease in inhibitory tone on pyramidal cells. ${ }^{147}$ The reduction in SICI thus indicates that synaptic GABAergic inhibition of pyramidal neurons may be reduced to some extent in the motor cortex. Moreover, a single oral dose of haloperidol $\left(\mathrm{D}_{2}\right.$ receptor antagonist and agonist of $\varsigma$ receptors) was shown to decrease SICI in healthy volunteers. ${ }^{148}$

The data for the effect of antipsychotic treatment on SICI are broadly suggestive that antipsychotic medications do not have a consistent impact on the reduced SICI observed in patients with schizophrenia. Other receptors that have been shown to play a role in modulating GABA-A receptor-mediated postsynaptic inhibition include presynaptic GABA-B autoreceptors, noradrenaline and $\varsigma$ receptor systems. ${ }^{29,77,90,149}$ Our results showed that reduced SICI is the most consistent finding among several studies using TMS to assess excitatory and inhibitory processes in schizophrenia. These results suggest impaired GABA-A-mediated cortical inhibition affecting the prodromal stage and persisting during the progression of the disease.

Most of the studies showed no statistically significant difference between patients and healthy controls in terms of motor threshold, in RMT or AMT (18 of 22 studies), MEP amplitude elicited by suprathreshold stimuli (7 of 9 studies) or ICF (8 of 9 studies). We also found no significant difference in the degree of LICI and SAI between patients and healthy controls, although only 1 study examined each measure. The results for the duration of CSP were inconclusive: of the 9 studies that delivered monophasic TMS pulses to the motor cortex and recorded responses from the surface of the hand muscle, $4^{43,86,93,95}$ detected no significant group difference (most or all patients were medicated; 6 patients were taking clozapine), $2^{82,96}$ reported shorter CSP duration in both medicated and 
unmedicated patients, and $3^{52,58,94}$ reported longer CSP duration in patients (most or all were medicated; 2 patients were taking clozapine). However, we found greater consistency in the data for the effect of antipsychotic treatment, especially clozapine, on increasing CSP duration. This result might have important implications for the treatment of schizophrenia, because GABA-B-mediated inhibition might be involved in some of the benefits induced by drugs.

Interestingly, it has been shown that mice with a GABA-B1 deficit exhibited marked hyperlocomotion when exposed to a novel environment. ${ }^{150}$ In addition, loss of GABA-B activity resulted in a sustained moderate hyperdopaminergic state, which was phenotypically revealed by contextual hyperlocomotor activity. Taken together, these results suggest an association between deficits in GABA-B neurotransmission and an altered dopaminergic system, which might provide insights for therapeutic intervention.

Furthermore, CSP duration increases with stimulus intensity in a sigmoid fashion, and the actual effect of positive modulators of GABA-A receptors on CSP may depend on whether measurement is obtained in the low- or high-intensity range of the sigmoid curve, implying that different underlying processes may be at work for different intensity ranges. ${ }^{19,151}$ Therefore, it is likely that antipsychotic treatment may interact with variations in stimulus intensity (from 120\% AMT to $90 \%$ maximum stimulator output) to explain some of the inconsistencies related to CSP duration that we found.

Neural oscillations represent an interplay between excitatory glutamatergic pyramidal neurons in the cortex and local circuit GABAergic interneurons (modulated by additional neurotransmitter systems such as dopamine, serotonin, noradrenaline, choline and metabotropic glutamate receptors) and thus may be particularly useful as indices of $\mathrm{E} / \mathrm{I}$ balance across mental disorders. ${ }^{152,153}$ TMS-elicited neural oscillations can provide such indices as oscillation inhibition and induction (or generation). Three studies ${ }^{99,105,106}$ assessed oscillations in the left primary motor cortex in schizophrenia using the LICI paradigm, indexing GABA-B receptor-mediated inhibition. Radhu and colleagues ${ }^{99}$ found significant within-group inhibition of both lower and higher frequencies in patients with schizophrenia and healthy controls, whereas Farzan and colleagues ${ }^{105}$ detected significant within-group inhibition of only $\delta, \theta$ and $\alpha$ bands in patients and healthy controls. One of the reasons for the difference might have been their choice of region of interest (i.e., multiple EEG channels versus a single channel). Nevertheless, all 3 studies reported that LICI induced a similar level of attenuation on all frequency bands between patients and healthy controls, whether the outcome measure was ERSP99,106 or area under the rectified curve for averaged EEG, ${ }^{105}$ suggesting that GABA-B receptor-mediated inhibition might be relatively intact in the motor cortex of patients with schizophrenia. This was consistent with findings that decreased expression of GABA-B receptor protein has been identified in the entorhinal cortex, hippocampus, inferior temporal cortex, prefrontal cortex and superior frontal cortex (Brodmann area 9) of the postmortem brains of people with schizophrenia, ${ }^{154-156}$ but to our knowledge so far similar findings have not been reported for GABA-B receptor protein in the motor cortex.
Taking together the results for SICI and LICI, there seem to be inhibitory deficits mediated by GABA-A receptors, but not GABA-B receptors in the motor cortex of patients with schizophrenia. It is worth noting that of the 3 studies above, only Radhu and colleagues ${ }^{106}$ corrected for the excitatory effect of the conditioning pulse that superimposes early EEG responses elicited by the test pulse (by subtracting the single pulse from the paired pulse lined up to the conditioning pulse). The other 2 studies did not correct for this confound. As well, although both LICI and CSP duration may reflect motor cortical GABA-B receptor-mediated postsynaptic inhibition, ${ }^{19}$ previous studies have shown differential modulation of LICI and CSP duration by drugs, disease and fatigue, which seems to imply that the mechanisms that underlie LICI and CSP are not identical ${ }^{29,157,158}$ and predictions for one cannot be made based on the other.

Two of the 3 studies that applied the LICI paradigm over the motor cortex reported oscillatory responses to singlepulse TMS stimuli in psychiatric patients and healthy controls. Farzan and colleagues ${ }^{105}$ reported no significant difference in area under the curve at channel C3 for any frequency after test pulses alone among patients with schizophrenia, patients with bipolar disorder and healthy controls. Ferrarelli and colleagues ${ }^{91}$ found no significant difference in global activation (global mean field power), amplitude change (ERSP) or intertrial phase synchrony (ITC) at any frequency after test pulses alone between patients with firstepisode psychosis and healthy controls, but the patients showed significantly lower relative spectral power in the $27 \sim 33 \mathrm{~Hz}$ range (high $\beta /$ low $\gamma$ ) in a cluster of frontocentral electrodes overlying the motor cortex. However, Ferrarelli and colleagues ${ }^{91}$ did not specify how they calculated relative spectral power except by saying that it was "the percentage of power in a given frequency." We do not know whether the relative spectral power reflected traditional evoked power or a combination of evoked and induced power (like the ERSP), so it was more difficult to unambiguously interpret the meaning of this measure. ${ }^{152}$

Previous studies have shown a deficit in oscillation induction in the motor cortex of patients with schizophrenia in response to actively initiated movement and passive stimulation by TMS. Compared to healthy controls, patients treated with antipsychotics and likely with chronic schizophrenia (based on their average age of 42) demonstrated reduced intertrial coherence preceding a self-initiated buttonpress over the contralateral motor cortex (measured at FC3 and FC5) in the $\gamma(36 \sim 45 \mathrm{~Hz})$ and $\beta(16 \sim 24 \mathrm{~Hz})$ bands. ${ }^{159}$ Prepress neural synchrony is thought to represent the corollary discharge. ${ }^{159}$ On the other hand, in Ferrarelli and colleagues, ${ }^{160}$ medicated patients with chronic schizophrenia (mean duration of illness 13 years) showed a slowing of natural frequency in the motor cortex compared to healthy controls after single-pulse TMS stimuli that corresponded to $110 \sim 115 \%$ RMT. The total ERSP (cumulated between 8-50 Hz and 20-300 ms) was also reduced in the patients. Data were calculated in the EEG channel closest to the TMS coil to reflect the activity of the targeted area. ${ }^{160}$ Considering the evidence from previous research, it is possible that 
GABA-A receptor-mediated inhibition is involved in the disturbance of the $\gamma$ - and $\beta$-band oscillations observed in the motor cortex of patients with schizophrenia. ${ }^{152,161,162}$

The first ${ }^{81}$ of 2 studies investigating the reaction of the premotor cortex to single-pulse TMS stimuli in patients with schizophrenia and healthy volunteers reported reduced amplitude (ERSP) and intertrial phase synchrony (ITC) of $\gamma$-band oscillations between 12 and $100 \mathrm{~ms}$ after TMS in the right premotor cortex of patients. In the second study, consistent with this finding that $\gamma$ oscillations may be impaired in the premotor cortex of patients, Canali and colleagues ${ }^{107}$ reported that between 20 and $300 \mathrm{~ms}$ after TMS, the left premotor cortex of patients with schizophrenia, bipolar disorder and major depressive disorder oscillated at slower frequencies $(11 \sim 27 \mathrm{~Hz}$, high $\alpha / \beta)$ relative to healthy controls (21 50 Hz, high $\beta / \gamma)$ in response to TMS perturbation, suggesting a deficit in evoked or induced higher-frequency oscillations in the premotor cortex of patients with schizophrenia.

The main findings of the 3 studies that assessed LICI of the dIPFC were that the inhibition of $\gamma$ oscillations by the LICI paradigm in schizophrenia was less effective than in healthy controls, regardless of whether the outcome measure was the area under the rectified curve for average $\mathrm{EEG}^{105}$ or ERSP, 99,106 as well, the inhibition of other frequency bands $(\delta, \theta, \alpha$ and $\beta)$ may also be impaired in schizophrenia. This was consistent with a finding of lower expression of GABA-B receptor subunits in the postmortem superior frontal cortex (Brodmann area 9) of patients with schizophrenia relative to healthy controls, suggesting that slow inhibitory signals mediated by metabotropic GABA-B receptors could be reduced in the dIPFC of patients with schizophrenia. ${ }^{154,155}$ Furthermore, patients with schizophrenia showed an impaired LICI of $\gamma$ oscillations (measured by area under the curve) and other frequency bands (measured by ERSP) compared to patients with bipolar disorder ${ }^{1,105}$ and obsessive-compulsive disorder, ${ }^{99}$ respectively, but no significant difference was found between the latter 2 groups of patients and healthy controls. This finding suggests that reduced suppression of neural oscillations by LICI in the dIPFC of patients with schizophrenia could be a specific endophenotype for the disorder.

Although disinhibition of the motor cortex has been a ubiquitous finding in psychiatric illnesses, independent of medication status, ${ }^{17,163,164}$ the overall pattern of these GABAergic deficits may differ across disorders with no specific pattern of inhibitory deficits for each disorder and little evidence for GABAergic deficits in the dIPFC across different psychiatric illnesses. The reported impairments in evoked and induced frontal oscillatory activity, especially in the $\gamma$ band, have been thought to contribute to deficits in cognition, such as working memory and cognitive control. ${ }^{109,165-167}$ Our findings show abnormalities in $\gamma$ oscillations in this clinical population, such as a deficit of GABA-B receptor-mediated inhibition of $\gamma$ induced by LICI over the dlPFC, ${ }^{105}$ and deficits in $\gamma$ synchronization over the premotor cortex. ${ }^{113}$ These findings might suggest selective $\gamma$ impairment in this population. It has been consistently shown that inhibition of spiking parvalbumin interneurons induces suppression of $\gamma$ oscillatory activity, ${ }^{168}$ and postmortem studies show reduction of GAD67 in this subclass of GABAergic interneurons in patients with schizophrenia. ${ }^{12}$ In addition, because cortical inhibition determines the spread of cortical activation along the cortical surface, ${ }^{169}$ the excessive propagation of TMS-induced oscillations (i.e., increased cortical conductivity) found in this population might be because of deficient cortical inhibition. ${ }^{85}$

The findings of the 2 studies by Noda and colleagues ${ }^{97,108}$ indicate that GABA-A receptor function (assessed by SICI) and the function of a low-threshold excitatory cortical circuit that may involve glutamatergic or noradrenergic receptor activation (assessed by ICF) may be impaired in the dlPFC of patients with schizophrenia relative to healthy controls. Central cholinergic activity (assessed by SAI) may also differ between patients and healthy controls because the paradigm induced differential modulation of N100 (in the dIPFC) and P180 (in the motor cortex) amplitudes in the 2 groups. SAI increased GABA-B receptor-mediated inhibition in healthy controls, but it produced the opposite effect in the dIPFC of patients. If $\mathrm{P} 180$ amplitude reflects neuronal membrane excitability, ${ }^{170,171}$ SAI made the neuronal membrane in the motor cortex of patients more excitable than that of healthy controls.

The GABA-B receptor-mediated inhibitory signals may be involved in modulating the evoked or induced oscillatory activity in the motor cortex and dIPFC. There are a few caveats related to the original studies included in the review: although 3 studies s9,105,106 $^{2}$ chose the Talairach coordinates of $(x, y, z=-50,30,36)$ and 2 studies $^{97,108}$ administered TMS paradigms at the F5 electrode site for stimulation of the dIPFC, stimulation of the premotor cortex was generally not associated with specific coordinates or electrode sites. Only 5 of 9 TMS-EEG studies ${ }^{81,85,91,105,107}$ used masking noise or sham TMS to control for the confounding effect of auditory-evoked potentials on the cortical EEG responses to TMS. Finally, all but 1 of the TMS-EEG studies tested fewer than 20 patients with schizophrenia, so the findings of these studies were based on relatively small sample sizes.

\section{Effect of antipsychotic medications on TMS-EMG and TMS-EEG measures}

Antipsychotic treatment seemed to have no effect on RMT, ${ }^{96,98,104,129}$ AMT, ${ }^{96,129}$ MEP amplitudes elicited by singlepulse suprathreshold stimuli (usually with an intensity that was set to produce $1 \mathrm{mV}$ MEPs), ${ }^{96,104,129} \mathrm{ICF}^{87,96,130,131}$ or LICI, ${ }^{98}$ indicating that the lack of difference observed between patients and healthy controls on these measures in the motor cortex was not likely to be due to the effect of antipsychotics. One study showed that medication may have reduced interhemispheric facilitation, but not inhibitory connectivity, between the left dorsal premotor cortex and the right motor cortex. ${ }^{129}$ The findings for medication effects on SICI were inconclusive; antipsychotic treatment seemed to enhance SICI in 1 study $^{130}$ but reduced it in another ${ }^{96}$ Interestingly, of the 4 studies that compared unmedicated patients to healthy controls, ${ }^{87,96,130,131} 3$ reported no significant difference in SICI between groups. ${ }^{87,96,131}$ Thus, the extent to which reduced SICI in medicated patients with chronic schizophrenia can be attributed to medication effect or chronic illness remains to be determined. 
Certain antipsychotics may enhance GABA-A receptormediated neurotransmission: olanzapine and haloperidol increased the density of benzodiazepine-sensitive GABA-A receptors in rat prefrontal cortex without altering the total population of GABA-A receptors after 28 days of drug infusion, suggesting that prolonged antipsychotic administration resulted in a "reshuffling" of GABA-A receptor subtypes. ${ }^{172}$ Acute administration of olanzapine and clozapine increased the neuroactive steroid allopregnanolone (a potent positive allosteric modulator of GABA-A receptors) in rat cerebral cortex in a dose-dependent manner. ${ }^{173}$ These studies demonstrate the potential of antipsychotics to influence GABA-A receptor function and hence SICI. SICI was not correlated with illness duration in patients with schizophrenia, ${ }^{45,60}$ although those who had a relatively short duration of illness (e.g., less than 3 years) or treatment (i.e., not longer than 6 weeks continuously) also showed reduced SICI in the motor cortex compared to healthy controls. ${ }^{46,60,174}$ Therefore, the decrease in SICI in schizophrenia may have been independent of illness and treatment duration.

As already mentioned, one of our most consistent findings was the effect of clozapine on CSP duration. This is noteworthy because of the unique place of clozapine in the antipsychotic armamentarium as the only medication licensed for use in treatment-refractory schizophrenia. Although it is clear that clozapine does not exert its unique effect via dopaminergic receptor antagonism (as observed in all other antipsychotic medications), its mechanism of action remains unclear. Clozapine was shown to increase CSP duration in patients with schizophrenia in 4 of 4 studies (2 were longitudinal). ${ }^{87,90,95,131}$ Therefore, the effect of clozapine on CSP duration suggests that clozapine may exert part of its antipsychotic effects by improving GABA-B-mediated inhibition. However, the current review did not find a significant correlation between CSP and symptom ratings, suggesting that further studies would be needed to clarify the role of GABA-B in treatment-refractory schizophrenia and the mechanism underlying clozapine's unique efficacy. ${ }^{6}$ Interestingly, clozapine had no effect on RMT, MEP amplitudes elicited by single-pulse suprathreshold stimuli, SICI or ICF. ${ }^{44,87,95}$

Other widely used antipsychotic medications include risperidone and olanzapine, and risperidone monotherapy also increased CSP duration with no effect on RMT in a beforeand-after treatment study with a group of medication-naive patients with first-episode schizophrenia. ${ }^{132}$ However, olanzapine and risperidone exerted differential effects on RMT in a cross-sectional study. ${ }^{133}$ Depending on the transcallosal inhibition paradigm, TMS stimulation can activate inhibitory interneurons in the contralateral motor cortex via a pathway across the corpus callosum, producing a silent period in the ipsilateral tonic hand muscle (single-pulse transcallosal inhibition), or it can inactivate the inhibitory neurons in the contralateral cortex (i.e., an anti-inhibitory effect), reducing the silent period in the ipsilateral tonic hand muscle (dual-pulse transcallosal inhibition). ${ }^{133}$ Compared to risperidone, treatment with olanzapine might have led to increased inhibitory activity and a stronger anti-inhibitory effect in the contralateral motor cortex during single-pulse and dual-pulse transcallosal inhibition paradigms, respectively. ${ }^{133}$ Also, although group comparisons (olanzapine v. risperidone v. healthy controls) in Fitzgerald and colleagues ${ }^{133}$ could not tell us whether olanzapine and risperidone monotherapy had changed SICI, ICF and CSP duration in patients, the lack of statistical difference between the 2 medication groups suggest that the antipsychotics might have a similar effect on the 3 TMS indices.

None of the TMS-EMG indices evaluated in this review was significantly correlated with antipsychotic dose, suggesting that if antipsychotic treatment had an effect on the indices it was not dose-dependent. Finally, antipsychotic medications seemed to have no effect on any of the TMS-EEG outcomes we evaluated, including both the oscillatory responses to single-pulse stimuli and the SAI, SICI, ICF and LICI effects on TMS-EEG activity. However, these findings came from only 8 studies that performed cross-sectional group comparisons (2 studies ${ }^{91,97}$ ) or correlational analyses between antipsychotic doses and TMS-EEG activity (6 studies $^{81,97,99,105-107}$ ). The differences in the study samples (e.g., patients with first-episode or chronic schizophrenia), stimulation paradigms and outcome measures could also have influenced EEG responses to TMS in these studies.

\section{Relationship between TMS measures and symptom severity}

The MEP amplitude elicited by suprathreshold single-pulse stimuli was positively correlated with PANSS negative subscale ratings in 2 studies. ${ }^{54,87} \mathrm{MEP}$ amplitude is thought to reflect trans-synaptic activation of corticospinal neurons through a complex network of excitatory circuits. ${ }^{19}$ Thus, this correlation suggests that increased excitability of this system might be associated with more severe negative symptoms. Our findings also reported significant associations between symptom severity and SICI and ICF.

ICF is a net facilitation: it reflects the activity of an excitatory motor cortical circuit that is distinct from the SICI network but is closely linked to its function. ${ }^{19}$ Hence, correlations with SICI and ICF can be discussed together: reduced SICI and increased ICF (i.e., less inhibition and more excitation) were associated with more severe positive symptoms; increased SICI and reduced facilitatory modulation of N100 amplitude by ICF (i.e., more inhibition and less excitation) were associated with less overall symptom severity; and decreased ICF was related to less general psychopathology and more negative symptoms. General psychopathology may reflect deficits in cognition such as disorientation, poor attention, lack of insight and active social avoidance. ${ }^{175}$ It seems that less inhibition and more excitation might contribute to greater positive symptoms, deficits in cognition and overall symptom severity. One study also found that decreased ICF was associated with greater negative symptoms. ${ }^{54}$ However, it should be noted that half of the studies did not find a significant correlation between PANSS scores and SICI ( 3 of 6 studies) $)^{60,84,96}$ and ICF ( 3 of 7 studies), ${ }^{84,96,190}$ indicating that the relationship between symptoms and TMS neurophysiology needs further research.

Most of the relevant studies (6 of 8$)^{84,95,96,100,130,132}$ found no correlation between CSP duration and symptom ratings as assessed with the PANSS. Moreover, 2 studies found that less 
severe negative symptoms were associated with more facilitatory connection between the left dorsal premotor cortex and right motor cortex, ${ }^{129}$ and between the right posterior parietal cortex and right motor cortex. ${ }^{104}$ Finally, 4 studies examined the correlations between TMS-elicited oscillations and symptom ratings. ${ }^{85,91,106,107}$ Because they assessed different aspects of TMS-evoked or -induced oscillations (i.e., propagation or global conductivity of signal power, frontal $\beta / \gamma$ power, inhibition of $\gamma$ and overall power by LICI, and natural frequency) in different brain regions (entire brain, motor cortex, dlPFC and premotor cortex, respectively; all in the sensor space) and different patient populations (firstepisode psychosis or chronic schizophrenia), it was difficult to ascribe any consistent effects.

The association between SICI and working memory performance, as assessed by different tasks, was a relatively consistent finding. Increased working memory capacity was related to increased SICI in the left motor cortex of medicated patients with recent-onset schizophrenia (duration of illness less than 3 years), and in the left dlPFC in medicated patients with chronic schizophrenia. ${ }^{60,108}$ As well, when data from patients and healthy controls were pooled, increased SICI in the motor cortex was associated with better working memory task accuracy on the 3-back condition of the $n$-back task. ${ }^{93}$ Taken together, these results might imply a relationship between impaired GABA-A receptor-mediated inhibition and working memory deficits in schizophrenia. As well, more enhancement in SICI in the motor cortex of medicated patients with chronic schizophrenia was associated with more improvement in performance on the delayed recall of words in a verbal learning test, ${ }^{90}$ which is a sensitive measure for the diagnosis of amnestic mild cognitive impairment and early Alzheimer disease. ${ }^{176}$ However, the increase in SICI in the same group of patients was related to less improvement in executive function, including task switching and response inhibition (assessed by the time taken to complete the wordcolour subtest of the Stroop test). ${ }^{90}$ These correlations are consistent with the relationship between $\gamma$ oscillations and working memory.

Lower GABA-B receptor-mediated inhibition (as reflected by a lower degree of LICI in 1 study $^{143}$ and reduced N100 amplitude in another ${ }^{97}$ ) in the left dlPFC might also contribute to poorer working memory capacity and executive function (assessed by the Stroop task and the Trail-Making Test) in patients with schizophrenia. ${ }^{108,143}$ Noda and colleagues ${ }^{97}$ also showed that central cholinergic activity induced by the SAI paradigm increased N100 amplitude in the dIPFC of healthy controls, whereas cholinergic activity might be impaired in patients with schizophrenia, leading to suppression of the N100 amplitude in the dlPFC.

Social cognition is closely related to the negative symptoms of schizophrenia and forms an important deficit that prevents people from engaging in social activities and work. A key aspect of social cognition is the ability to infer the intentions of other people, and the mirror neuron system has been proposed to underpin this important function. Mirror neurons are neurons that fire both when we act and when we observe the same action performed by others. ${ }^{177}$ Mirror neuron activity has been indexed by increased MEP amplitude in response to suprathreshold single-pulse stimuli (with or without a reduced SICI and increased ICF) while observing an action. ${ }^{78,145,146}$ Three studies ${ }^{76,145,146}$ demonstrated that the mirror neuron system may be impaired in the motor cortex of patients with schizophrenia during action observation, whether the action is embedded in a social context or not. This suggests that an impaired mirror neuron system may be a potential marker of poorer social cognition and warrants further investigation for its effect on social networks and functioning.

\section{Relationship between TMS measures and illness duration}

The TMS-EMG and TMS-EEG measures evaluated the current review were not associated with duration of illness in schizophrenia, except in 1 study, ${ }^{104}$ which reported a significant correlation between illness duration and facilitatory connection between the right posterior parietal and right motor cortices in a group of patients with chronic schizophrenia (70\% of whom were medicated). Thus, the findings of this review do not support the premise that illness duration may itself induce physiologic changes in the brain.

\section{TMS-elicited plasticity and effective connectivity in schizophrenia}

In medicated patients with chronic schizophrenia relative to healthy controls, prolonged duration of transcallosal inhibition evoked by the single-pulse transcallosal inhibition paradigm, indexing functional connectivity in schizophrenia, may hint at functional impairment of transcallosal motor pathways in schizophrenia that might result from structural changes of the relevant callosal fibres. ${ }^{79,80,178}$ The facilitatory parietomotor connection in the right hemisphere may also be reduced in both medicated and unmedicated patients with chronic schizophrenia. ${ }^{104}$ One study ${ }^{81}$ showed that signal propagation of TMS-elicited neural activity in patients with schizophrenia (most of whom were medicated) was limited to the premotor and motor areas along the midline in both hemispheres in the first $100 \mathrm{~ms}$ after TMS of the right premotor cortex, suggesting defective interactions among cortical areas in chronic schizophrenia. Another study ${ }^{83}$ found that higher average global voltage 400 750 ms after TMS of the left motor cortex in patients with chronic schizophrenia (most of whom were medicated) may be driven by increased total power in the $\delta$ and $\beta$ or $\gamma$ bands between 400 800 ms in many EEG channels. Such prolonged activation relative to healthy controls may suggest increased interhemispheric functional connectivity between homologous motor areas and extended to bilateral temporoparietal regions in patients with schizophrenia. ${ }^{85}$ These studies showed that functional connectivity in patients with chronic schizophrenia may be disrupted in both time and space (including interhemispheric and within-hemisphere connections). This finding supports a contemporary theory that schizophrenia symptoms arise as a consequence of functional neural dysconnectivity rather than because of a specific regional deficit or lesion. ${ }^{179}$ 
Deficient neural plasticity was reported in 4 studies that assessed motor cortical plasticity in patients with schizophrenia. ${ }^{44,45,89,96}$ Patients with chronic schizophrenia (most of whom were medicated) in Strube and colleagues ${ }^{89}$ showed a deficit in focal long-term potentiation-like plasticity induced by paired associative stimulation, which emulates spike-timing-dependent plasticity. ${ }^{52,180}$ Another study ${ }^{43}$ reported deficient anodal tDCSinduced nonfocal long-term potentiation-like plasticity in patients with multiple psychotic episodes (most of them medicated) but not in those with a single episode (recent-onset schizophrenia; most of them medicated), suggesting "a neurodegenerative process of cortical plasticity in patients with schizophrenia." However, a recent meta-analysis reported that illness duration might not influence motor cortical plasticity in schizophrenia, and that altered plasticity may predate the manifestation of clinical symptoms. ${ }^{181}$ Furthermore, Hasan and colleagues ${ }^{45}$ speculated that patients with chronic schizophrenia might have disturbed GABAergic neurotransmission because tDCS enhanced SICI only in those with recent-onset schizophrenia. Still, no significant difference in SICI was found among those with recent-onset schizophrenia, multi-episode schizophrenia or healthy controls before tDCS administration in that study, and SICI was not correlated with the number of psychotic episodes or duration of psychosis in patients with schizophrenia. The third study ${ }^{96}$ found that a 15-minute train of low-frequency repetitive TMS increased RMT and decreased CSP duration in healthy controls but not in medicated and unmedicated patients with chronic schizophrenia; however, the change in MEP amplitudes did not differ significantly among groups when controlling for multiple comparisons. The fourth study ${ }^{131}$ demonstrated that medicated and unmedicated patients had an equal deficit in orienting post-training thumb movements in the direction of training compared to healthy controls, indicating that the mechanisms involved in use-dependent reorganization of cortical synaptic connections may be disrupted in schizophrenia.

Although the studies used change in MEP amplitude to index plasticity, it is important to note that before the administration of the plasticity paradigms (i.e., paired associative stimulation, anodal tDCS and rTMS), there was no significant difference in MEP amplitude among their respective participant groups, implying that the excitability of the motor cortex may not differ between patients and healthy controls. This may suggest that dysfunction may arise only as a consequence of dynamic change secondary to increased load or demand placed on the neural connectivity in the system. Indeed, tDCS to enhance neural plasticity and connectivity has been a feature of more recent novel interventions to improve cognitive symptoms in schizophrenia. ${ }^{182}$ Both NMDA receptor function and dopaminergic transmission have been implicated as essential components in long-term potentiation and use-dependent motor learning; GABAergic and cholinergic activity may also play a role in modulating plasticity. ${ }^{45,89,131}$

\section{Future research}

It is clear that in the current diagnostic classification system there are many symptoms that cross diagnostic boundaries. It is likely that these symptoms will be underpinned by some common pathophysiological processes; ${ }^{183}$ for example, reduced working memory and decreased SICI might be a common deficit for patients with schizophrenia, attention-deficit/ hyperactivity disorder, amnestic mild cognitive impairment and early Alzheimer disease. ${ }^{163,176}$ Therefore, this review has implications beyond schizophrenia, because patients with other mental health diagnoses are also likely to have distinct E/I balance characteristics, as measured by EMG and EEG responses to standardized TMS probes. ${ }^{99,105,163}$ TMS-EMG and TMS-EEG offer an opportunity to develop a novel and improved understanding of the physiologic processes that underlie mental disorders and assess the therapeutic effect of antipsychotic medications (and novel treatment approaches).

Specific applications that would benefit from this approach would be to use TMS-EMG and TMS-EEG to help predict disease progression from prodrome to first-episode psychosis, to full-blown schizophrenia. Several studies have addressed this question by making cross-sectional group comparisons involving medication-naive patients with prodromal symptoms, patients with first-episode psychosis (or schizophrenia) and healthy controls. ${ }^{45,58,91,174,184}$ The findings from these studies and the studies in patients with chronic schizophrenia seem to suggest decreased SICI across the illness stages. However, decreased SICI is not sufficient in itself to predict illness progression. Another key mechanism for this approach would be to better understand the E/I balance and its implications for mechanisms that underlie treatment response in schizophrenia, aiding in the selection of drug development and fulfilling an urgent need for novel therapies for treatment-resistant schizophrenia.

\section{Risk of bias}

For the subscales "measurement of exposure" and "blinding of outcome assessments," details about blinding during data collection and analysis were not available in most of the studies and we were unable to judge whether the presumed absence of blinding in these studies may have affected the results. For the assessment of "selective outcome reporting," we did not find the preregistered experimental protocol for any of the included studies, so formal assessment was not possible and we based our judgment on whether the outcomes and analyses defined in the hypotheses and methods sections (particularly the statistical analyses) were described in the results section of the studies.

\section{Limitations}

We aimed to review the literature on TMS-EMG and TMSEEG in schizophrenia. The included studies were heterogeneous with respect to TMS paradigms and outcome measures, research questions and analyses, and participant samples, precluding a meta-analysis of the findings. Moreover, although the current review was set to include only those with a diagnosis of schizophrenia or schizoaffective disorder, one of the included studies ${ }^{91}$ examined patients with first-episode psychosis. As well, the various studies adopted different methods and criteria for excluding participants who might 
also have had substance abuse. Some studies used urine tests but others did not, and the time periods for which substance use was excluded before study participation differed. One of the studies ${ }^{83}$ screened the healthy controls for substance abuse and we assumed they did the same for patients with schizophrenia. Most of the patients included in the current review were medicated and had chronic schizophrenia. Most of the studies used cross-sectional group comparisons and had small patient sample sizes (less than 30). Longitudinal studies with larger samples may be needed to investigate changes in the brain associated with treatment effects and disease progression by following up participants from prodrome to schizophrenia diagnosis, or even longer.

\section{Conclusion}

Previous systematic reviews have reviewed either TMS-EMG ${ }^{17}$ or TMS-EEG ${ }^{185}$ studies that investigated the neurophysiology of schizophrenia. The current review combined the literature for TMS-EMG and TMS-EEG in schizophrenia, and it gauged the influence of antipsychotic medications, symptom severity and illness duration on TMS-EMG and TMS-EEG activity. Furthermore, we selected only studies that included participants without documented substance abuse to reduce the confounding effects of illicit drug use on brain activity. However, this review focused on patients with schizophrenia or schizoaffective disorder, and not on other populations such as people at risk of developing psychosis (also called prodromal) or patients with first-episode psychosis. Therefore, it is not suitable for answering questions about the neurophysiology of the subclinical or early stages of the psychosis spectrum.

Our findings indicate reduced SICI and relatively intact LICI in the motor cortex of patients with schizophrenia, whereas both SICI and LICI may be reduced in the dlPFC. Notably, the findings of a LICI deficit in the dIPFC of patients with chronic schizophrenia might be specific to schizophrenia; its diagnostic value warrants further investigation. As well, glutamatergic or noradrenergic and cholinergic activity might also be disrupted in the dlPFC in schizophrenia, as assessed by ICF and SAI paradigms, respectively. Furthermore, previous electrophysiological evidence (with and without TMS) suggests that evoked or induced higher-frequency $(\beta / \gamma)$ oscillations may be impaired in the motor, premotor and dorsolateral prefrontal cortices of patients with schizophrenia.

Clozapine prolonged CSP duration, and olanzapine and risperidone may exert similar effects on CSP. Improved working memory performance was associated with enhanced SICI in the left motor cortex and the dIPFC, and also with increased GABA-B receptor-mediated inhibition (as measured by LICI and N100 amplitude) in the left dlPFC, linking working memory performance to GABAergic activity in the left motor cortex and dlPFC. Moreover, schizophrenia may induce widespread deficits in brain functional connectivity in both time and space, as well as deficient motor cortical plasticity. These findings suggest that TMS coupled with EMG and EEG have the potential to be used as biomarkers for illness and treatment in schizophrenia. Future TMS-EEG studies would need to verify the reproducibility of these early findings, preferably using standardized experimental methods, including procedures to properly mask the auditory and somatosensory input caused by TMS to minimize their contribution to TMS-EEG responses.

Acknowledgements: S. Shergill and I. Premoli were supported by the National Institute for Health Research (NIHR) Maudsley Biomedical Research Centre at South London and Maudsley NHS Foundation Trust and King's College London. S. Shergill was also supported by MRC Grant 400790. Fiona Watson is a library and archives manager at the Royal College of Psychiatrists, and she helped create the search terms.

Affiliations: From the Department of Psychosis Studies, Institute of Psychiatry, Psychology and Neuroscience, King's College London, London, UK (Hou, Shergill); the Department of Basic and Clinical Neuroscience, Institute of Psychiatry, Psychology and Neuroscience, King's College London, London, UK (Santoro, Biondi, Premoli); and the Kent and Medway Medical School, Canterbury, UK (Shergill).

Competing interests: None declared

Contributors: V. Santoro, A. Biondi, S. Shergill and I. Premoli designed the study. M. Hou acquired the data, which I Premoli analyzed. M. Hou, V. Santoro and I. Premoli wrote the article, which A. Biondi, S. Shergill and I. Premoli reviewed. All authors approved the final version to be published and can certify that no other individuals not listed as authors have made substantial contributions to the paper.

Content licence: This is an Open Access article distributed in accordance with the terms of the Creative Commons Attribution (CC BY-NC-ND 4.0) licence, which permits use, distribution and reproduction in any medium, provided that the original publication is properly cited, the use is noncommercial (i.e., research or educational use), and no modifications or adaptations are made. See: https://creativecommons.org/licenses/by-nc-nd/4.0/

Data sharing: All data are contained within the paper.

\section{References}

1. McGrath J, Saha S, Chant D, et al. Schizophrenia: a concise overview of incidence, prevalence, and mortality. Epidemiol Rev 2008;30:67-76.

2. Van Os J, Kapur S. Schizophrenia. Lancet 2009;374:635-45.

3. Lieberman JA, Fenton WS. Delayed detection of psychosis: causes, consequences, and effect on public health. Am J Psychiatry 2000;157:1727-30.

4. Lieberman JA, Perkins D, Belger A, et al. The early stages of schizophrenia: speculations on pathogenesis, pathophysiology, and therapeutic approaches. Biol Psychiatry 2001;50:884-97.

5. Murray RM, Lewis SW. Is schizophrenia a neurodevelopmental disorder? Br Med J (Clin Res Ed) 1987;295:681-2.

6. Rogasch NC, Daskalakis ZJ, Fitzgerald PB. Cortical inhibition, excitation, and connectivity in schizophrenia: a review of insights from transcranial magnetic stimulation. Schizophr Bull 2014;40:685-96.

7. Kane JM. The current status of neuroleptic therapy. J Clin Psychiatry 1989;50:322-8.

8. Coyle JT. Glutamate and schizophrenia: beyond the dopamine hypothesis. Cell Mol Neurobiol 2006;26:365-84.

9. Heresco-Levy U. Glutamatergic neurotransmission modulators as emerging new drugs for schizophrenia. Expert Opin Emerg Drugs 2005; 10:827-44.

10. Koster LS, Carbon M, Correll CU. Emerging drugs for schizophrenia: an update. Expert Opin Emerg Drugs 2014;19:511-31.

11. Gonzalez-Burgos G, Lewis DA. NMDA receptor hypofunction, parvalbumin-positive neurons, and cortical gamma oscillations in schizophrenia. Schizophr Bull 2012;38:950-7.

12. Hashimoto T, Volk DW, Eggan SM, et al. Gene expression deficits in a subclass of GABA neurons in the prefrontal cortex of subjects with schizophrenia. J Neurosci 2003;23:6315-26.

13. Homayoun H, Moghaddam B. NMDA receptor hypofunction produces opposite effects on prefrontal cortex interneurons and pyramidal neurons. J Neurosci 2007;27:11496-500. 
14. Lewis DA, Curley AA, Glausier JR, et al. Cortical parvalbumin interneurons and cognitive dysfunction in schizophrenia. Trends Neurosci 2012;35:57-67.

15. Lodge DJ, Behrens MM, Grace AA. A loss of parvalbumin-containing interneurons is associated with diminished oscillatory activity in an animal model of schizophrenia. J Neurosci 2009;29:2344-54.

16. Taylor SF, Tso IF. GABA abnormalities in schizophrenia: a methodological review of in vivo studies. Schizophr Res 2015;167:84-90.

17. Bunse T, Wobrock T, Strube W, et al. Motor cortical excitability assessed by transcranial magnetic stimulation in psychiatric disorders: a systematic review. Brain Stimul 2014;7:158-69.

18. Rothwell JC, Hallett M, Berardelli A, et al. Magnetic stimulation: motor evoked potentials. The International Federation of Clinical Neurophysiology. Electroencephalogr Clin Neurophysiol Suppl 1999; 52:97-103.

19. Ziemann U, Reis J, Schwenkreis P, et al. TMS and drugs revisited 2014. Clin Neurophysiol 2015;126:1847-68.

20. Awiszus F. TMS and threshold hunting. Suppl Clin Neurophysiol 2003;56:13-23.

21. Borckardt JJ, Nahas Z, Koola J, et al. Estimating resting motor thresholds in transcranial magnetic stimulation research and practice: a computer simulation evaluation of best methods. J ECT 2006;22:169-75.

22. Rossini PM, Berardelli A, Deuschl G, et al. Applications of magnetic cortical stimulation. The International Federation of Clinical Neurophysiology. Electroencephalogr Clin Neurophysiol Suppl 1999; 52:171-85.

23. Siebner HR, Ziemann U. What is the threshold for developing and applying optimized procedures to determine the corticomotor threshold? Clin Neurophysiol 2014;125:1-2.

24. Fuhr P, Agostino R, Hallett M. Spinal motor neuron excitability during the silent period after cortical stimulation. Electroencephalogr Clin Neurophysiol 1991;81:257-62.

25. Inghilleri M, Berardelli A, Cruccu G, et al. Silent period evoked by transcranial stimulation of the human cortex and cervicomedullary junction. J Physiol 1993;466:521-34.

26. Ziemann U, Netz J, Szelenyi A, et al. Spinal and supraspinal mechanisms contribute to the silent period in the contracting soleus muscle after transcranial magnetic stimulation of human motor cortex. Neurosci Lett 1993;156:167-71.

27. Hallett M. Transcranial magnetic stimulation: a primer. Neuron 2007;55:187-99.

28. Di Lazzaro V, Pilato F, Dileone M, et al. Segregating two inhibitory circuits in human motor cortex at the level of GABAA receptor subtypes: a TMS study. Clin Neurophysiol 2007;118:2207-14.

29. McDonnell MN, Orekhov Y, Ziemann U. The role of GABA(B) receptors in intracortical inhibition in the human motor cortex. Exp Brain Res 2006;173:86-93.

30. Boroojerdi B, Battaglia F, Muellbacher W, et al. Mechanisms influencing stimulus-response properties of the human corticospinal system. Clin Neurophysiol 2001;112:931-7.

31. Mohammadi B, Krampfl K, Petri S, et al. Selective and nonselective benzodiazepine agonists have different effects on motor cortex excitability. Muscle Nerve 2006;33:778-84.

32. Ziemann U, Chen R, Cohen LG, et al. Dextromethorphan decreases the excitability of the human motor cortex. Neurology 1998;51:1320-4.

33. Ziemann U, Tergau F, Wischer S, et al. Pharmacological control of facilitatory I-wave interaction in the human motor cortex. A paired transcranial magnetic stimulation study. Electroencephalogr Clin Neurophysiol 1998;109:321-30.

34. Ferbert A, Priori A, Rothwell JC, et al. Interhemispheric inhibition of the human motor cortex. J Physiol 1992;453:525-46.

35. Gerloff C, Cohen LG, Floeter MK, et al. Inhibitory influence of the ipsilateral motor cortex on responses to stimulation of the human cortex and pyramidal tract. J Physiol 1998;510(Pt 1):249-59.

36. Ilmoniemi RJ, Virtanen J, Ruohonen J, et al. Neuronal responses to magnetic stimulation reveal cortical reactivity and connectivity. Neuroreport 1997;8:3537-40.

37. Massimini M, Ferrarelli F, Huber R, et al. Breakdown of cortical effective connectivity during sleep. Science 2005;309:2228-32.

38. Paus T, Sipila PK, Strafella AP. Synchronization of neuronal activity in the human primary motor cortex by transcranial magnetic stimulation: an EEG study. J Neurophysiol 2001;86:1983-90.

39. Tremblay S, Rogasch NC, Premoli I, et al. Clinical utility and prospective of TMS-EEG. Clin Neurophysiol 2019;130:802-44.
40. Darmani G, Zipser CM, Bohmer GM, et al. Effects of the selective alpha5-GABAAR antagonist S44819 on excitability in the human brain: a TMS-EMG and TMS-EEG phase I study. J Neurosci 2016; 36:12312-20.

41. Premoli I, Castellanos N, Rivolta D, et al. TMS-EEG signatures of GABAergic neurotransmission in the human cortex. J Neurosci 2014;34:5603-12.

42. Premoli I, Bergmann TO, Fecchio M, et al. The impact of GABAergic drugs on TMS-induced brain oscillations in human motor cortex. Neuroimage 2017;163:1-12.

43. Pascual-Leone A, Amedi A, Fregni F, et al. The plastic human brain cortex. Annu Rev Neurosci 2005;28:377-401.

44. Daskalakis ZJ, Christensen BK, Fitzgerald PB, et al. Dysfunctional neural plasticity in patients with schizophrenia. Arch Gen Psychiatry 2008;65:378-85.

45. Hasan A, Nitsche MA, Rein B, et al. Dysfunctional long-term potentiation-like plasticity in schizophrenia revealed by transcranial direct current stimulation. Behav Brain Res 2011;224:15-22.

46. Hasan A, Schneider M, Schneider-Axmann T, et al. A similar but distinctive pattern of impaired cortical excitability in first-episode schizophrenia and ADHD. Neuropsychobiology 2013;67:74-83.

47. Randic M, Jiang MC, Cerne R. Long-term potentiation and longterm depression of primary afferent neurotransmission in the rat spinal cord. J Neurosci 1993;13:5228-41.

48. Bindman LJ, Lippold OC, Redfearn JW. Long-lasting changes in the level of the electrical activity of the cerebral cortex produced by polarizing currents. Nature 1962;196:584-5.

49. Nitsche MA, Paulus W. Excitability changes induced in the human motor cortex by weak transcranial direct current stimulation. J Physiol 2000;527:633-9.

50. Purpura DP, McMurtry JG. Intracellular activities and evoked potential changes during polarization of motor cortex. J Neurophysiol 1965;28:166-85.

51. Stagg CJ, Nitsche MA. Physiological basis of transcranial direct current stimulation. Neuroscientist 2011;17:37-53.

52. Classen J, et al. Paired associative stimulation. Suppl Clin Neurophysiol 2004:57:563-9.

53. Kim SY, Park JE, Jae Lee Y, et al. Testing a tool for assessing the risk of bias for nonrandomized studies showed moderate reliability and promising validity. J Clin Epidemiol 2013;66:408-14.

54. Tang Y, Zhang T, Edelman B, et al. Prolonged cortical silent period among drug-naive subjects at ultra-high risk of psychosis. Schizophr Res 2014;160:124-30.

55. Fusar-Poli $\mathrm{P}$, Borgwardt $\mathrm{S}$, Bechdolf $\mathrm{A}$, et al. The psychosis highrisk state: a comprehensive state-of-the-art review. JAMA Psychiatry 2013;70:107-20.

56. McGlashan T, Miller T, Woods S, et al. Structured interview for prodromal syndromes (SIPS). New Haven (CT): Yale University; 2001.

57. Miller TJ, McGlashan TH, Rosen JL, et al. Prodromal assessment with the structured interview for prodromal syndromes and the scale of prodromal symptoms: predictive validity, interrater reliability, and training to reliability. Schizophr Bull 2003;29:703.

58. Hasan A, Wobrock T, Grefkes C, et al. Deficient inhibitory cortical networks in antipsychotic-naive subjects at risk of developing first-episode psychosis and first-episode schizophrenia patients: a cross-sectional study. Biol Psychiatry 2012;72:744-51.

59. Bechdolf A, Müller H, Stützer H, et al. Rationale and baseline characteristics of PREVENT: a second-generation intervention trial in subjects at-risk (prodromal) of developing first-episode psychosis evaluating cognitive behavior therapy, aripiprazole, and placebo for the prevention of psychosis. Schizophr Bull 2011;37:S111-21.

60. Takahashi S, Ukai S, Kose A, et al. Reduction of cortical GABAergic inhibition correlates with working memory impairment in recent onset schizophrenia. Schizophr Res 2013;146:238-43.

61. Lang N, Rothkegel H, Peckolt H, et al. Effects of lacosamide and carbamazepine on human motor cortex excitability: a doubleblind, placebo-controlled transcranial magnetic stimulation study. Seizure 2013;22:726-30.

62. Menzler K, Hermsen A, Balkenhol K, et al. A common SCN 1 A splice-site polymorphism modifies the effect of carbamazepine on cortical excitability-a pharmacogenetic transcranial magnetic stimulation study. Epilepsia 2014;55:362-9.

63. Ziemann U, Lonnecker S, Steinhoff BJ, et al. Effects of antiepileptic drugs on motor cortex excitability in humans: a transcranial magnetic stimulation study. Ann Neurol 1996;40:367-78. 
64. Ilic TV, Korchounov A, Ziemann U. Methylphenidate facilitates and disinhibits the motor cortex in intact humans. Neuroreport 2003;14:773-6.

65. Gerdelat-Mas A, Loubinoux I, Tombari D, et al. Chronic administration of selective serotonin reuptake inhibitor (SSRI) paroxetine modulates human motor cortex excitability in healthy subjects. Neuroimage 2005;27:314-22.

66. Paulus W, Classen J, Cohen LG, et al. State of the art: pharmacologic effects on cortical excitability measures tested by transcranial magnetic stimulation. Brain Stimul 2008;1:151-63.

67. Di Lazzaro V, Oliviero A, Meglio M, et al. Direct demonstration of the effect of lorazepam on the excitability of the human motor cortex. Clin Neurophysiol 2000;111:794-9.

68. Inghilleri M, Berardelli A, Marchetti $\mathrm{P}$, et al. Effects of diazepam, baclofen and thiopental on the silent period evoked by transcranial magnetic stimulation in humans. Exp Brain Res 1996;109:467-72.

69. Siebner HR, Dressnandt J, Auer C, et al. Continuous intrathecal baclofen infusions induced a marked increase of the transcranially evoked silent period in a patient with generalized dystonia. Muscle Nerve 1998;21:1209-12.

70. Di Lazzaro V, Oliviero A, Pilato F, et al. Neurophysiological predictors of long term response to AChE inhibitors in AD patients. J Neurol Neurosurg Psychiatry 2005;76:1064-9.

71. Di Lazzaro V, Pilato F, Dileone M, et al. Dissociated effects of diazepam and lorazepam on short-latency afferent inhibition. J Physiol 2005;569:315-23.

72. Meyer BU, Roricht S, Woiciechowsky C. Topography of fibers in the human corpus callosum mediating interhemispheric inhibition between the motor cortices. Ann Neurol 1998;43:360-9.

73. Di Lazzaro V, Pilato F, Dileone M, et al. Segregating two inhibitory circuits in human motor cortex at the level of GABAA receptor subtypes: a TMS study. Clin Neurophysiol 2007;118:2207-14.

74. Di Lazzaro V, Pilato F, Dileone M, et al. GABAA receptor subtype specific enhancement of inhibition in human motor cortex. J Physiol 2006;575:721-6.

75. Ziemann U, Lonnecker S, Steinhoff BJ, et al. The effect of lorazepam on the motor cortical excitability in man. Exp Brain Res 1996; 109:127-35

76. Pierantozzi M, Marciani MG, Giuseppina Palmieri M, et al. Effect of vigabatrin on motor responses to transcranial magnetic stimulation: an effective tool to investigate in vivo GABAergic cortical inhibition in humans. Brain Res 2004;1028:1-8.

77. Werhahn KJ, Kunesch E, Noachtar S, et al. Differential effects on motorcortical inhibition induced by blockade of GABA uptake in humans. J Physiol 1999;517:591-7.

78. Bagewadi VI, Meherwan Mehta U, Nakik SS, et al. Diminished modulation of motor cortical reactivity during context-based action observation in schizophrenia. Schizophr Res 2019;204:222-9.

79. Bajbouj M, Gallinat J, Niehaus L, et al. Abnormalities of inhibitory neuronal mechanisms in the motor cortex of patients with schizophrenia. Pharmacopsychiatry 2004;37:74-80.

80. Boroojerdi B, Topper R, Foltys H, et al. Transcallosal inhibition and motor conduction studies in patients with schizophrenia using transcranial magnetic stimulation. Br J Psychiatry 1999;175: 375-9.

81. Du X, Choa FS, Chiappelli J, et al. Aberrant middle prefrontalmotor cortex connectivity mediates motor inhibitory biomarker in schizophrenia. Biol Psychiatry 2019;85:49-59.

82. Du X, Hong LE. Test-retest reliability of short-interval intracortical inhibition and intracortical facilitation in patients with schizophrenia. Psychiatry Res 2018;267:575-81.

83. Ferrarelli F, Massimini M, Peterson MJ, et al. Reduced evoked gamma oscillations in the frontal cortex in schizophrenia patients: a TMS/EEG study. Am J Psychiatry 2008;165:996-1005.

84. Fitzgerald PB, Brown TL, Daskalakis ZJ, et al. A transcranial magnetic stimulation study of inhibitory deficits in the motor cortex in patients with schizophrenia. Psychiatry Res 2002;114:11-22.

85. Frantseva M, Cui J, Farzan F, et al. Disrupted cortical conductivity in schizophrenia: TMS-EEG study. Cereb Cortex 2014;24:211-21.

86. Lindberg PG, Térémetz M, Charron S, et al. Altered cortical processing of motor inhibition in schizophrenia. Cortex 2016;85:1-12.

87. Liu SK, Fitzgerald PB, Daigle M, et al. The relationship between cortical inhibition, antipsychotic treatment, and the symptoms of schizophrenia. Biol Psychiatry 2009;65:503-9.
88. Reid PD, Daniels B, Rybak M, et al. Cortical excitability of psychiatric disorders: reduced post-exercise facilitation in depression compared to schizophrenia and controls. Aust N Z J Psychiatry 2002;36:669-73

89. Strube W, Bunse T, Nitsche MA, et al. Differential response to anodal tDCS and PAS is indicative of impaired focal LTP-like plasticity in schizophrenia. Behav Brain Res 2016;311:46-53.

90. Yildiz MI, Temucin C, Ertugrul A. [The relationship of the change in symptoms and cognitive functions with the change in cortical inhibition parameters measured by transcranial magnetic stimulation: an eight-week follow-up study]. Turk Psikiyatri Derg 2015;26:161-71.

91. Ferrarelli F, Kaskie RE, Graziano B, et al. Abnormalities in the evoked frontal oscillatory activity of first-episode psychosis: a TMS/EEG study. Schizophr Res 2019;206:436-9.

92. Chroni E, Lekka NP, Tsoussis I, et al. Effect of exercise on motor evoked potentials elicited by transcranial magnetic stimulation in psychiatric patients. J Clin Neurophysiol 2002;19:240-4.

93. Bridgman AC, Barr MS, Goodman MS, et al. Deficits in GABAA receptor function and working memory in non-smokers with schizophrenia. Schizophr Res 2016;171:125-30.

94. Soubasi E, Chroni E, Gourzis P, et al. Cortical motor neurophysiology of patients with schizophrenia: a study using transcranial magnetic stimulation. Psychiatry Res 2010;176:132-6.

95. Kaster TS, de Jesus D, Radhu N, et al. Clozapine potentiation of GABA mediated cortical inhibition in treatment resistant schizophrenia. Schizophr Res 2015;165:157-62.

96. Fitzgerald PB, Brown TL, Marston NAU, et al. Reduced plastic brain responses in schizophrenia: a transcranial magnetic stimulation study. Schizophr Res 2004;71:17-26.

97. Noda Y, Zomorrodi R, Backhouse F, et al. Reduced short-latency afferent inhibition in prefrontal but not motor cortex and its association with executive function in schizophrenia: a combined TMSEEG study. Schizophr Bull 2018;44:193-202.

98. Fitzgerald PB, Brown TL, Marston NAU, et al. A transcranial magnetic stimulation study of abnormal cortical inhibition in schizophrenia. Psychiatry Res 2003;118:197-207.

99. Radhu N, Dominguez LG, Farzan F, et al. Evidence for inhibitory deficits in the prefrontal cortex in schizophrenia. Brain 2015;138: 483-97.

100. Ahlgren-Rimpilainen A, Lauerma H, Kahkonen S, et al. Disrupted central inhibition after transcranial magnetic stimulation of motor cortex in schizophrenia with long-term antipsychotic treatment. ISRN Psychiatry 2013;2013:876171.

101. Tokimura H, Lazzaro VDi, Tokimura $Y$, et al. Short latency inhibition of human hand motor cortex by somatosensory input from the hand. J Physiol 2000;523:503-13.

102. Ilic TV, Meintzschel F, Cleff U, et al. Short-interval paired-pulse inhibition and facilitation of human motor cortex: the dimension of stimulus intensity. J Physiol 2002;545:153-67.

103. Kujirai T, Caramia MD, Rothwell JC, et al. Corticocortical inhibition in human motor cortex. J Physiol 1993;471:501-19.

104. Koch G, Ribolsi M, Mori F, et al. Connectivity between posterior parietal cortex and ipsilateral motor cortex is altered in schizophrenia. Biol Psychiatry 2008;64:815-9.

105. Farzan F, Barr MS, Levinson AJ, et al. Evidence for gamma inhibition deficits in the dorsolateral prefrontal cortex of patients with schizophrenia. Brain 2010;133:1505-14.

106. Radhu N, Dominguez LG, Greenwood TA, et al. Investigating cortical inhibition in first-degree relatives and probands in schizophrenia. Sci Rep 2017;7:43629.

107. Canali P, Sarasso S, Rosanova M, et al. Shared reduction of oscillatory natural frequencies in bipolar disorder, major depressive disorder and schizophrenia. I Affect Disord 2015;184:111-5.

108. Noda Y, Barr MS, Zomorrodi R, et al. Evaluation of short interval cortical inhibition and intracortical facilitation from the dorsolateral prefrontal cortex in patients with schizophrenia. Sci Rep 2017; 7:17106.

109. Cho RY, Konecky RO, Carter CS. Impairments in frontal cortical gamma synchrony and cognitive control in schizophrenia. Proc Natl Acad Sci U S A 2006;103:19878-83.

110. Light GA, Hsu JL, Hsieh MH, et al. Gamma band oscillations reveal neural network cortical coherence dysfunction in schizophrenia patients. Biol Psychiatry 2006;60:1231-40.

111. Spencer KM, Nestor PG, Niznikiewicz MA, et al. Abnormal neural synchrony in schizophrenia. J Neurosci 2003;23:7407-11. 
112. Daskalakis ZJ, Farzan F, Barr MS, et al. Long-interval cortical inhibition from the dorsolateral prefrontal cortex: a TMS-EEG study. Neuropsychopharmacology 2008;33:2860-9.

113. Ferrarelli F, Massimini M, Peterson MJ, et al. Reduced evoked gamma oscillations in the frontal cortex in schizophrenia patients: a TMS/EEG study. Am J Psychiatry 2008;165:996-1005.

114. Haracz JL. Neural plasticity in schizophrenia. Schizophr Bull 1985; 11:191-229.

115. Nitsche MA, Paulus W. Sustained excitability elevations induced by transcranial DC motor cortex stimulation in humans. Neurology 2001;57:1899-901.

116. Nitsche MA, Nitsche MS, Klein CC, et al. Level of action of cathodal DC polarisation induced inhibition of the human motor cortex. Clin Neurophysiol 2003;114:600-4.

117. Nitsche MA, Roth A, Kuo MF, et al. Timing-dependent modulation of associative plasticity by general network excitability in the human motor cortex. J Neurosci 2007;27:3807-12.

118. Stefan K, Kunesch E, Cohen LG, et al. Induction of plasticity in the human motor cortex by paired associative stimulation. Brain 2000;123:572-84.

119. Lally J, MacCabe JH. Antipsychotic medication in schizophrenia: a review. Br Med Bull 2015;114:169-79.

120. Ginovart N, Kapur S. Role of dopamine $D(2)$ receptors for antipsychotic activity. Handb Exp Pharmacol 2012;212:27-52.

121. Sakai K, Gao XM, Hashimoto T, et al. Traditional and new antipsychotic drugs differentially alter neurotransmission markers in basal ganglia-thalamocortical neural pathways. Synapse 2001; 39:152-60.

122. Bakshi VP, Geyer MA. Antagonism of phencyclidine-induced deficits in prepulse inhibition by the putative atypical antipsychotic olanzapine. Psychopharmacology (Berl) 1995;122:198-201.

123. Moore NA. Olanzapine: preclinical pharmacology and recent findings. Br J Psychiatry Suppl 1999;37:41-4.

124. McQueen G, Sendt KV, Gillespie A, et al. Changes in brain glutamate on switching to clozapine in treatment-resistant schizophrenia. Schizophr Bull 2021;47:662-71.

125. Kapur S, Seeman P. Antipsychotic agents differ in how fast they come off the dopamine D2 receptors. Implications for atypical antipsychotic action. J Psychiatry Neurosci 2000;25:161-6.

126. Meltzer HY. Treatment of the neuroleptic-nonresponsive schizophrenic patient. Schizophr Bull 1992;18:515-42.

127. Kapur S, Zipursky R, Jones C, et al. Relationship between dopamine D2 occupancy, clinical response, and side effects: a doubleblind PET study of first-episode schizophrenia. Am J Psychiatry 2000;157:514-20.

128. Egerton A, Bhachu A, Merritt K, et al. Effects of antipsychotic administration on brain glutamate in schizophrenia: a systematic review of longitudinal (1)H-MRS studies. Front Psychiatry 2017;8:66.

129. Ribolsi M, Mori F, Magni V, et al. Impaired inter-hemispheric facilitatory connectivity in schizophrenia. Clin Neurophysiol 2011;122:512-7.

130. Daskalakis ZJ, Christensen BK, Chen R, et al. Evidence for impaired cortical inhibition in schizophrenia using transcranial magnetic stimulation. Arch Gen Psychiatry 2002;59:347-54.

131. Daskalakis ZJ, Christensen BK, Fitzgerald PB, et al. Increased cortical inhibition in persons with schizophrenia treated with clozapine. J Psychopharmacol 2008;22:203-9.

132. Ustohal L, Mayerova M, Hublova V, et al. Risperidone increases the cortical silent period in drug-naive patients with first-episode schizophrenia: a transcranial magnetic stimulation study. J Psychopharmacol 2017;31:500-4.

133. Fitzgerald PB, Brown TL, Daskalakis ZJ, et al. A transcranial magnetic stimulation study of the effects of olanzapine and risperidone on motor cortical excitability in patients with schizophrenia. Psychopharmacology (Berl) 2002;162:74-81.

134. Kay SR, Fiszbein A, Opler LA. The positive and negative syndrome scale (PANSS) for schizophrenia. Schizophr Bull 1987;13:261-76.

135. Andreasen NC. Scale for the assessment of positive symptoms (SAPS). Iowa City (IA): University of Iowa; 1984.

136. Andreasen NC. Negative symptoms in schizophrenia. Definition and reliability. Arch Gen Psychiatry 1982;39:784-8.

137. Overall JE, Gorham DR. The brief psychiatric rating scale. Psychol Rep 1962;10:799-812.

138. Endicott J, Spitzer RL, Fleiss JL, et al. The global assessment scale. A procedure for measuring overall severity of psychiatric disturbance. Arch Gen Psychiatry 1976;33:766-71.
139. Diagnostic and statistical manual of mental disorders. 4th ed. Washington (DC): American Psychiatric Association; 1994.

140. Thomas M, Szentgyorgyi T, Vanes LD, et al. Cognitive performance in early, treatment-resistant psychosis patients: could cognitive control play a role in persistent symptoms? Psychiatry Res 2021;295:113607.

141. Barch DM, Ceaser A. Cognition in schizophrenia: core psychological and neural mechanisms. Trends Cogn Sci 2012;16:27-34.

142. Keefe RS, Goldberg TE, Harvey PD, et al. The Brief Assessment of Cognition in Schizophrenia: reliability, sensitivity, and comparison with a standard neurocognitive battery. Schizophr Res 2004:68:283-97.

143. Lett TA, Kennedy JL, Radhu N, et al. Prefrontal white matter structure mediates the influence of gad1 on working memory. Neuropsychopharmacology 2016;41:2224-31.

144. Mehta UM, Thirthalli J, Basavaraju R, et al. Association of intracortical inhibition with social cognition deficits in schizophrenia: findings from a transcranial magnetic stimulation study. Schizophr Res 2014;158:146-50.

145. Mehta UM, Thirthalli J, Basavaraju R, et al. Reduced mirror neuron activity in schizophrenia and its association with theory of mind deficits: evidence from a transcranial magnetic stimulation study. Schizophr Bull 2014;40:1083-94.

146. Basavaraju R, Mehta UM, Thirthalli J, et al. Mirror neuron dysfunction and ego-boundary disturbances in schizophrenia: a transcranial magnetic stimulation study. Indian J Psychol Med 2015;37: 58-65.

147. de Jonge JC, Vinkers $\mathrm{CH}$, Hulshoff Pol HE, et al. GABAergic mechanisms in schizophrenia: linking postmortem and in vivo studies. Front Psychiatry 2017;8:118.

148. Ziemann U, Tergau F, Bruns D, et al. Changes in human motor cortex excitability induced by dopaminergic and anti-dopaminergic drugs. Electroencephalogr Clin Neurophysiol 1997;105:430-7.

149. Gilbert DL, Ridel KR, Sallee FR, et al. Comparison of the inhibitory and excitatory effects of ADHD medications methylphenidate and atomoxetine on motor cortex. Neuropsychopharmacology 2006;31: 442-9.

150. Vacher CM, Gassmann M, Desrayaud S, et al. Hyperdopaminergia and altered locomotor activity in GABAB1-deficient mice. J Neurochem 2006:97:979-91.

151. Kimiskidis VK, Papagiannopoulos S, Sotirakoglou K, et al. Silent period to transcranial magnetic stimulation: construction and properties of stimulus-response curves in healthy volunteers. Exp Brain Res 2005;163:21-31.

152. Javitt DC, Siegel SJ, Spencer KM, et al. A roadmap for development of neuro-oscillations as translational biomarkers for treatment development in neuropsychopharmacology. Neuropsychopharmacology 2020;45:1411-22.

153. Uhlhaas PJ, Haenschel C, Nikolic D, et al. The role of oscillations and synchrony in cortical networks and their putative relevance for the pathophysiology of schizophrenia. Schizophr Bull 2008;34 927-43.

154. Fatemi SH, Folsom TD, Thuras PD. Deficits in GABA(B) receptor system in schizophrenia and mood disorders: a postmortem study. Schizophr Res 2011;128:37-43.

155. Fatemi SH, Folsom TD, Thuras PD. GABAA and GABAB receptor dysregulation in superior frontal cortex of subjects with schizophrenia and bipolar disorder. Synapse 2017;71:e21973.

156. Kantrowitz J, Citrome L, Javitt D. GABA(B) receptors, schizophrenia and sleep dysfunction: a review of the relationship and its potential clinical and therapeutic implications. CNS Drugs 2009;23:681-91.

157. Benwell NM, Mastaglia FL, Thickbroom GW. Differential changes in long-interval intracortical inhibition and silent period duration during fatiguing hand exercise. Exp Brain Res 2007;179:255-62.

158. Poston B, Kukke SN, Paine RW, et al. Cortical silent period duration and its implications for surround inhibition of a hand muscle. Eur J Neurosci 2012;36:2964-71.

159. Ford JM, Roach BJ, Faustman WO, et al. Out-of-synch and out-ofsorts: dysfunction of motor-sensory communication in schizophrenia. Biol Psychiatry 2008;63:736-43.

160. Ferrarelli F, Sarasso S, Guller Y, et al. Reduced natural oscillatory frequency of frontal thalamocortical circuits in schizophrenia. Arch Gen Psychiatry 2012;69:766-74.

161. Bartos M, Vida I, Jonas P. Synaptic mechanisms of synchronized gamma oscillations in inhibitory interneuron networks. Nat Rev Neurosci 2007:8:45-56. 
162. Buzsaki G, Wang XJ. Mechanisms of gamma oscillations. Annu Rev Neurosci 2012;35:203-25.

163. Radhu N, de Jesus DR, Ravindran LN, et al. A meta-analysis of cortical inhibition and excitability using transcranial magnetic stimulation in psychiatric disorders. Clin Neurophysiol 2013;124: 1309-20.

164. Radhu N, Ravindran LN, Levinson AJ, et al. Inhibition of the cortex using transcranial magnetic stimulation in psychiatric populations: current and future directions. J Psychiatry Neurosci 2012;37:369-78.

165. Chen CM, Stanford AD, Mao X, et al. GABA level, gamma oscillation, and working memory performance in schizophrenia. Neuroimage Clin 2014;4:531-9.

166. Haenschel C, Bittner RA, Waltz J, et al. Cortical oscillatory activity is critical for working memory as revealed by deficits in early-onset schizophrenia. J Neurosci 2009;29:9481-9.

167. Minzenberg MJ, Firl AJ, Yoon JH, et al. Gamma oscillatory power is impaired during cognitive control independent of medication status in first-episode schizophrenia. Neuropsychopharmacology 2010;35:2590-9.

168. Sohal VS, Zhang F, Yizhar O, et al. Parvalbumin neurons and gamma rhythms enhance cortical circuit performance. Nature 2009; 459:698-702.

169. Llinás R, Urbano FJ, Leznik E, et al. Rhythmic and dysrhythmic thalamocortical dynamics: GABA systems and the edge effect. Trends Neurosci 2005;28:325-33.

170. Darmani G, Bergmann TO, Zipser C, et al. Effects of antiepileptic drugs on cortical excitability in humans: a TMS-EMG and TMSEEG study. Hum Brain Mapp 2019;40:1276-89.

171. Premoli I, Costantini A, Rivolta D, et al. The effect of lamotrigine and levetiracetam on TMS-evoked EEG responses depends on stimulation intensity. Front Neurosci 2017;11:585.

172. Skilbeck KJ, O'Reilly JN, Johnston GA, et al. The effects of antipsychotic drugs on GABAA receptor binding depend on period of drug treatment and binding site examined. Schizophr Res 2007:90:76-80

173. Marx CE, VanDoren MJ, Duncan GE, et al. Olanzapine and clozapine increase the GABAergic neuroactive steroid allopregnanolone in rodents. Neuropsychopharmacology 2003;28:1-13.
174. Wobrock T, Schneider-Axmann T, Retz W, et al. Motor circuit abnormalities in first-episode schizophrenia assessed with transcranial magnetic stimulation. Pharmacopsychiatry 2009;42:194-201.

175. Shankar G, Nate C. Positive and Negative Syndrome Scale as a long-term outcome measurement tool in patients receiving clozapine ODT - a pilot study. Pharm Pract (Granada) 2007;5:42-5.

176. Zhao Q, Lv Y, Zhou Y, et al. Short-term delayed recall of auditory verbal learning test is equivalent to long-term delayed recall for identifying amnestic mild cognitive impairment. PLoS One 2012;7:e51157.

177. Acharya S, Shukla S. Mirror neurons: enigma of the metaphysical modular brain. J Nat Sci Biol Med 2012;3:118-24.

178. Vanes LD, Mouchlianitis E, Barry E, et al. Cognitive correlates of abnormal myelination in psychosis. Sci Rep 2019;9:1-9.

179. Stephan KE, Friston KJ, Frith CD. Dysconnection in schizophrenia: from abnormal synaptic plasticity to failures of self-monitoring. Schizophr Bull 2009;35:509-27.

180. Muller-Dahlhaus F, Ziemann U, Classen J. Plasticity resembling spike-timing dependent synaptic plasticity: the evidence in human cortex. Front Synaptic Neurosci 2010;2:34.

181. Mehta UM, Thanki MV, Padmanabhan J, et al. Motor cortical plasticity in schizophrenia: a meta-analysis of transcranial magnetic stimulation-electromyography studies. Schizophr Res 2019;207: 37-47.

182. Orlov ND, Tracy DK, Joyce D, et al. Stimulating cognition in schizophrenia: a controlled pilot study of the effects of prefrontal transcranial direct current stimulation upon memory and learning. Brain Stimul 2017;10:560-6.

183. Insel T, Cuthbert B, Garvey M, et al. Research domain criteria (RDoC): toward a new classification framework for research on mental disorders. Am J Psychiatry 2010;167:748-51.

184. Hasan A, Falkai P, Wobrock T. Transcranial brain stimulation in schizophrenia: targeting cortical excitability, connectivity and plasticity. Curr Med Chem 2013;20:405-13.

185. Li X, Honda S, Nakajima S, et al. TMS-EEG research to elucidate the pathophysiological neural bases in patients with schizophrenia: a systematic review. J Pers Med 2021;11:388. 